\title{
Stability analysis and control of the flow in a symmetric channel with a sudden expansion
}

Andrea Fani, Simone Camarri, and Maria Vittoria Salvetti

Citation: Phys. Fluids 24, 084102 (2012); doi: 10.1063/1.4745190

View online: http://dx.doi.org/10.1063/1.4745190

View Table of Contents: http://pof.aip.org/resource/1/PHFLE6/v24/i8

Published by the American Institute of Physics.

\section{Related Articles}

Heat flow control in thermo-magnetic convective systems using engineered magnetic fields

Appl. Phys. Lett. 101, 123507 (2012)

Computation of effective free surfaces in two phase flows

Phys. Fluids 24, 087101 (2012)

The dynamics of liquid drops and their interaction with solids of varying wettabilities

Phys. Fluids 24, 082001 (2012)

Note: Pneumatically modulated liquid delivery with feedback control

Rev. Sci. Instrum. 83, 076102 (2012)

Using sharp transitions in contact angle hysteresis to move, deflect, and sort droplets on a superhydrophobic surface

Phys. Fluids 24, 062001 (2012)

\section{Additional information on Phys. Fluids}

Journal Homepage: http://pof.aip.org/

Journal Information: http://pof.aip.org/about/about_the_journal

Top downloads: http://pof.aip.org/features/most_downloaded

Information for Authors: http://pof.aip.org/authors

\section{ADVERTISEMENT}

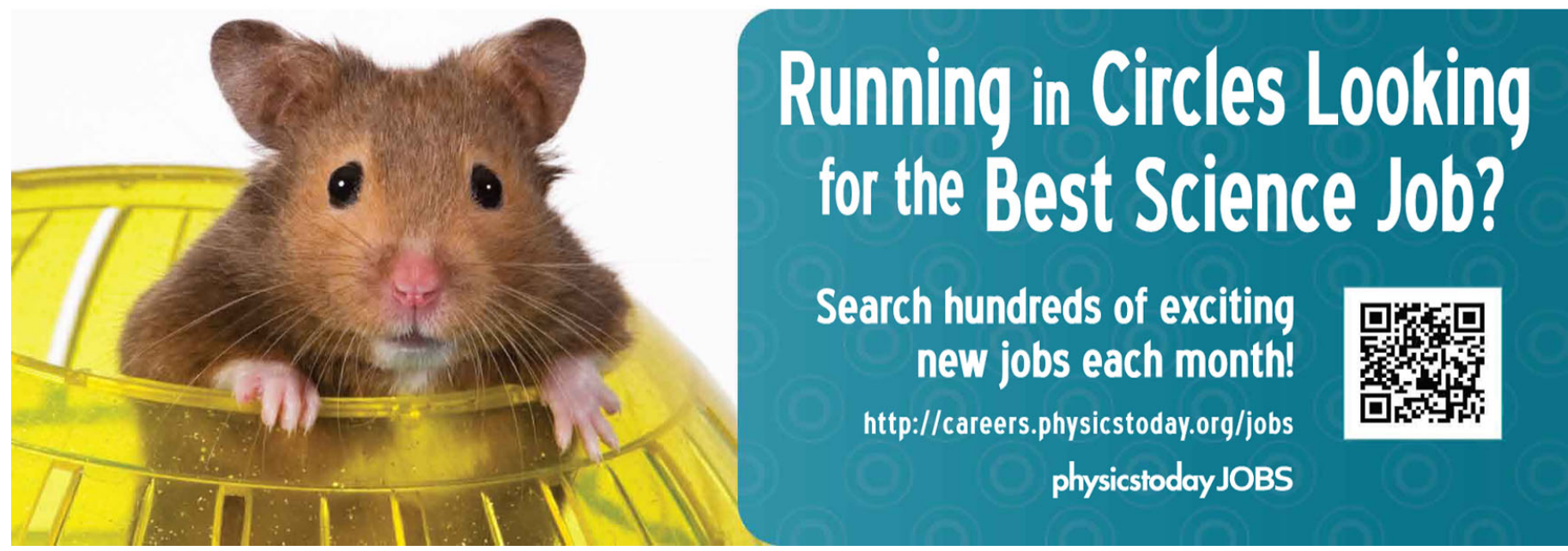




\title{
Stability analysis and control of the flow in a symmetric channel with a sudden expansion
}

\author{
Andrea Fani, Simone Camarri, ${ }^{\text {a) }}$ and Maria Vittoria Salvetti \\ Dipartimento di Ingegneria Aerospaziale, Università di Pisa, via G. Caruso 8, \\ 56122 Pisa, Italy
}

(Received 20 December 2011; accepted 26 July 2012; published online 16 August 2012)

\begin{abstract}
The laminar flow in two-dimensional diffusers may produce either symmetric or nonsymmetric steady solutions, depending on the value of the Reynolds number as compared with some critical value. The stability properties of the flow are studied in the context of linear theory. In this context, a sensitivity analysis of the flow instability is carried out with respect to perturbations that may be produced by a realistic passive control, thus providing qualitative hints and quantitative information for the control design. Following the so-obtained information, a passive control is built by introducing a small cylinder in the flow with the aim of stabilizing the unstable symmetric flow configuration in the diffuser. The effectiveness of this control is finally assessed by direct numerical simulation. It is shown that the introduction of the cylinder, placed following the indications of the linear sensitivity analysis in the stable asymmetric flow configuration, allows a steady completely symmetric or less asymmetric flow to be recovered. The flow transient between the uncontrolled asymmetric solution and the symmetric controlled one is analyzed in terms of streamlines and vorticity evolution; the effects of the cylinder introduction on flow dissipation are also assessed. (c) 2012 American Institute of Physics. [http://dx.doi.org/10.1063/1.4745190]
\end{abstract}

\section{INTRODUCTION}

The two-dimensional flow in a plane channel with a symmetric sudden expansion is a basic flow configuration characterized by a symmetry breaking bifurcation. This intriguing physics of the flow and its importance in engineering applications have attracted considerable previous attention. Experimental and numerical studies (see, e.g., Durst et al., ${ }^{1,2}$ Cherdron et al., ${ }^{3}$ and Fearn et al. ${ }^{4}$ ) investigated the considered flow in case of moderate expansion ratio (ER), defined as the ratio between the height of the channel after and before the expansion. It was found that there exists a critical value of the Reynolds number, $R e_{c}$, such that, when $R e<R e_{c}$ the flow in the channel is steady, two-dimensional and symmetric, and when $R e>R e_{c}$ the flow is still two-dimensional and steady, but it becomes asymmetric. The value of $R e_{c}$ is shown to decrease as the expansion ratio increases in Battaglia et al. ${ }^{5}$ and Drikakis. ${ }^{6}$ The physical mechanism originating the bifurcation has been numerically investigated in Hawa and Rusak $^{7}$ and Alleborn et al. ${ }^{8}$ by asymptotic techniques. The same authors Hawa and $\operatorname{Rusak}^{9}$ show that the described pitchfork bifurcation changes nature as a small asymmetry of the channel is considered, leading to two disconnected branches of equilibrium states, one describing a gradual and stable change with the Reynolds number, and the other one containing a large-amplitude stable state and a small-amplitude unstable state. The asymmetric case is also considered in Alleborn et al. ${ }^{8}$ Three-dimensional effects on the considered flow have been investigated in Chiang et al. ${ }^{10}$ and Battaglia and Papadopoulos. ${ }^{11}$

In this study, we consider a value of the expansion ratio equal to $E R=3$ as, for instance, in Hawa and Rusak ${ }^{7}$ and Battaglia et al. ${ }^{5}$ A 2D linear stability analysis of the symmetric steady solution in the considered configuration has shown that for $R e>R e_{c}$, one real unstable mode exists which

\footnotetext{
a)s.camarri@ing.unipi.it.
} 
drives the solution to an asymmetric state (see, e.g., Refs. 5,6, and 7). The aim of the present paper is to use the linear stability results and related sensitivity analysis approaches, to design a realistic flow control strategy, aimed at stabilizing the symmetric steady solution by the introduction of a small fixed cylinder in the flow.

We first verified that the analysis can be limited to the two-dimensional case, through an investigation of the linear stability of the considered target flow to three-dimensional perturbations, reported in the Appendix of the present paper, which showed that the most unstable mode is indeed two-dimensional.

As for the sensitivity analysis, two techniques recently proposed in the literature (Giannetti and Luchini ${ }^{12}$ and Marquet et al. ${ }^{13}$ ) are our starting points. Giannetti and Luchini ${ }^{12}$ proposed the guidelines to investigate the sensitivity of the unstable mode, and of the related instability, with respect to a structural perturbation of the linearized flow equations. In their analysis the base flow is kept unchanged, while a forcing term is added in the equations describing the evolution of the flow perturbation with respect to the base flow. On the other hand, the sensitivity of the instability with respect to a perturbation of the base flow was addressed in Marquet et al. ${ }^{13}$ In the present paper, these techniques are applied for the first time to the flow inside a channel with a sudden expansion, in order to add information on the characteristics of the instability in this flow configuration and to obtain indications useful for a generic control method of this flow. Indeed, the regions of the flow where the instability originates and where it is necessary to act for its control are identified.

Moving forward, we combine and specialize these two techniques in a realistic control strategy, based on the introduction of a small cylinder in the flow. To this purpose the effect of the controlling cylinder is modeled by the introduction of a steady pointwise drag force, whose amplitude is directly related to the cylinder diameter. The cylinder introduction produces a modification of the base flow and also of the linearized perturbation problem. Therefore, the analyses described in Giannetti and Luchini $^{12}$ and Marquet et al. ${ }^{13}$ are used to estimate the effect of the cylinder on the unstable mode, giving quantitative information for the design of the control. This leads to sensitivity maps which give combinations of diameter/position of the control cylinder which may stabilize the flow, according to the adopted simplified model of the cylinder effect.

When the cylinder is modeled as a pointwise forcing and the analysis is carried out linearizing the equations around the unperturbed flow, it is important to characterize the range of validity of the linearized analysis as the forcing amplitude is increased. This characterization is carried out here by comparing the results of the linearized analysis to the stability properties of the flow obtained when a finite-amplitude forcing is introduced. This indication is also preliminary to the use of iterative techniques for the control design as the one described in Camarri and Iollo. ${ }^{14}$

Finally, direct numerical simulations (DNS), in which the cylinder is physically introduced in the flow following the indications of the previous sensitivity analyses, are reported in the present paper. The aim of these simulations is threefold. First, this is a verification that a real control method can be built from the previously described sensitivity analyses. In particular, DNS provides a validation of the procedure followed for its design, which was based on several approximations. To the author knowledge, this final assessment is rarely carried out in the works documented in the literature. Second, the robustness of the proposed control is checked by investigating its capability of recovering the symmetric solution starting from the stable asymmetric one and its behavior when applied to a slightly asymmetric channel configuration, as considered in Hawa and Rusak. ${ }^{9}$ Finally, the information available from DNS is used to provide a physical interpretation for the success of the proposed control, which is built, as previously described, by a theoretical/mathematical procedure.

\section{FLOW CONFIGURATION AND METHODOLOGY}

The incompressible flow in a 2D-channel with a symmetric sudden expansion is considered here. The flow configuration and the used frame of reference are reported in Fig. 1. With reference to Fig. 1, the expansion rate is $\mathrm{ER}=D / d=3$. The incoming flow is assumed to have a Poiseuille profile and the Reynolds number is defined as $R e=U_{c} d / \nu$, where $U_{c}$ is the maximum velocity of the 


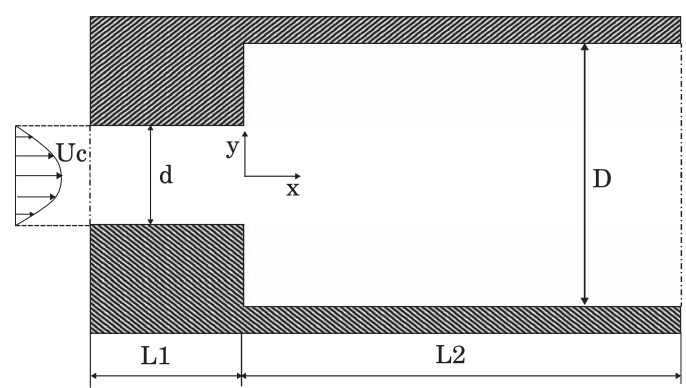

FIG. 1. Channel geometry.

Poiseuille profile. All the quantities shown throughout the paper are non-dimensional. The velocity $U_{c}$ and height $d$ are used as reference velocity and length scales.

The fluid motion is described by the two-dimensional unsteady incompressible Navier-Stokes equations,

$$
\begin{aligned}
& \frac{\partial \mathbf{U}}{\partial t}+\mathbf{U} \cdot \nabla \mathbf{U}+\nabla P-\frac{1}{R e} \nabla^{2} \mathbf{U}=\mathbf{0}, \\
& \nabla \cdot \mathbf{U}=0,
\end{aligned}
$$

where $\mathbf{U}$ is the velocity vector with components $\mathbf{U}=(U, V)$ and $P$ is the reduced pressure. No-slip boundary conditions are imposed at the lateral walls, while at the inlet a Poiseuille velocity profile of the following form is assumed: $U=-4(y-1 / 2)(y+1 / 2), V=0$. Finally, free outflow conditions of the following type are used: $P-R e^{-1} \partial_{x} U=0, \partial_{x} V=0$.

\section{A. Linear stability analysis}

The instability onset is studied using linear theory and a normal-mode analysis. The flow quantities are decomposed in a steady (base flow) part and in an unsteady perturbation as

$$
\begin{aligned}
& \mathbf{U}(x, y, t)=\mathbf{U}_{b}(x, y)+\epsilon \mathbf{u}(x, y, t), \\
& P(x, y, t)=P_{b}(x, y)+\epsilon p(x, y, t),
\end{aligned}
$$

where the amplitude $\epsilon$ is assumed to be small. The choice of two-dimensional perturbations, instead of three-dimensional ones, is justified in the Appendix, where it is shown that the flow under investigation is stable with respect to three-dimensional perturbations in the range of considered Reynolds numbers.

Substituting Eqs. (2) into Eqs. (1) and linearizing with respect to $\epsilon$, we obtain the following equations, describing the evolution of the linear perturbations,

$$
\begin{aligned}
& \frac{\partial \mathbf{u}}{\partial t}+\mathbf{L}\left(\mathbf{U}_{b}, R e\right) \mathbf{u}+\nabla p=\mathbf{0} \\
& \nabla \cdot \mathbf{u}=0
\end{aligned}
$$

where

$$
\mathbf{L}\left(\mathbf{U}_{b}, R e\right) \mathbf{u}=\mathbf{u} \cdot \nabla \mathbf{U}_{b}+\mathbf{U}_{\mathbf{b}} \cdot \nabla \mathbf{u}-\frac{1}{R e} \nabla^{2} \mathbf{u} .
$$

The steady incompressible Navier-Stokes equations governing the base flow are therefore

$$
\begin{aligned}
& \mathbf{U}_{b} \cdot \nabla \mathbf{U}_{b}+\nabla P_{b}-\frac{1}{R e} \nabla^{2} \mathbf{U}_{b}=0, \\
& \nabla \cdot \mathbf{U}_{b}=0,
\end{aligned}
$$


with the same boundary conditions as those used for problem (1). Since the base flow is a steady and symmetric solution, it is possible to solve problem (5) in just half of the domain, i.e., where $y>0$, by imposing the symmetry condition $\partial_{y} U_{b}=0, V_{b}=0$ on the centerline $y=0$.

The perturbation is sought in the form of normal modes $\mathbf{q}(x, y, t)=(\mathbf{u}, p)(x, y, t)$ $=(\hat{\mathbf{u}}, \hat{p})(x, y) \exp (\sigma t)$, where $\sigma$ is a generally complex eigenvalue associated with the complex fields $(\hat{\mathbf{u}}, \hat{p})$. Equations (3) become

$$
\begin{aligned}
& \sigma \hat{\mathbf{u}}+\hat{\mathbf{u}} \cdot \nabla \mathbf{U}_{b}+\mathbf{U}_{\mathbf{b}} \cdot \nabla \hat{\mathbf{u}}+\nabla \hat{p}-\frac{1}{R e} \nabla^{2} \hat{\mathbf{u}}=\mathbf{0}, \\
& \nabla \cdot \hat{\mathbf{u}}=0 .
\end{aligned}
$$

The boundary conditions associated with (6) are: $\hat{\mathbf{u}}=0$ at the inlet and on the walls and free outflow conditions at the outlet. Equations (6) along with the boundary conditions are an eigenvalue problem. The flow is linearly stable when all the problem eigenvalues, $\sigma=\lambda+i \omega$, are characterized by $\lambda<0$.

\section{B. Sensitivity to a structural perturbation of the linearized NS equations}

In Giannetti and Luchini ${ }^{12}$ it is shown by a perturbation analysis that, for each considered global mode $(\hat{\mathbf{u}}, \hat{p}, \sigma)$, the addition of a perturbation $\delta \hat{\mathbf{f}}$ at the r.h.s. of the momentum equation (6a) causes the following variation $\delta \sigma$ of the eigenvalue $\sigma$ :

$$
\delta \sigma=\frac{\left(\hat{\mathbf{u}}^{+}, \delta \hat{\mathbf{f}}\right)}{\left(\hat{\mathbf{u}}^{+}, \hat{\mathbf{u}}\right)},
$$

where $(a, b)=\int_{\Omega}\left(a^{*} \cdot b\right) d \Omega$ is the scalar product between the complex vectors $a$ and $b$ on the flow domain $\Omega$ and the asterisk $*$ denotes the conjugate of a complex quantity. The vector field $\hat{\mathbf{u}}^{+}$is the velocity field of the mode adjoint to $(\hat{\mathbf{u}}, \hat{p}, \sigma)$, solution of the following eigenvalue problem:

$$
\begin{aligned}
& \sigma^{*} \hat{\mathbf{u}}^{+}+\nabla \mathbf{U}_{b} \cdot \hat{\mathbf{u}}^{+}-\mathbf{U}_{\mathbf{b}} \cdot \nabla \hat{\mathbf{u}}^{+}+\nabla \hat{p}^{+}-\frac{1}{R e} \nabla^{2} \hat{\mathbf{u}}^{+}=\mathbf{0} \\
& \nabla \cdot \hat{\mathbf{u}}^{+}=0 .
\end{aligned}
$$

The boundary conditions associated with problem (8) are: $\hat{\mathbf{u}}^{+}=0$ at the inlet and on the lateral walls, and $p^{+} \mathbf{n}-R e^{-1}\left(\mathbf{n} \cdot \nabla \hat{\mathbf{u}}^{+}\right)=\left(\mathbf{U}_{\mathbf{b}} \cdot \mathbf{n}\right) \hat{\mathbf{u}}^{+}$at the outlet (see Marquet et al. ${ }^{13}$ ), $\mathbf{n}$ being the normal unit vector pointing outside the flow domain.

In the present work, we use the following condition to normalize the adjoint velocity field:

$$
\left(\hat{\mathbf{u}}^{+}, \hat{\mathbf{u}}\right)=1 .
$$

Giannetti and Luchini ${ }^{12}$ have considered the effect of a small modification of the evolution operator under the form of a localized force-velocity coupling, which can also be loosely interpreted as a linear feedback induced by an actuator located at the same station as the sensor, both placed in a a generic point within the flow domain. In particular, Giannetti and Luchini ${ }^{12}$ have considered a structural perturbation with the following form:

$$
\delta \hat{\mathbf{f}}=C_{0} \cdot \hat{\mathbf{u}} \delta\left(x-x_{0}, y-y_{0}\right),
$$

where $C_{0}$ is a generic constant (feedback) matrix, the symbol · stands for matrix vector product, $\left(x_{0}, y_{0}\right)$ are the coordinates of the point where the feedback acts, and $\delta(x, y)$ denotes the Dirac delta function. For the structural perturbation (10) Giannetti and Luchini ${ }^{12}$ have shown that

$$
\left\|\delta \sigma\left(x_{0}, y_{0}\right)\right\| \leq C_{0} \cdot\left\|\hat{\mathbf{u}}^{+}\left(x_{0}, y_{0}\right)\right\| \cdot\left\|\hat{\mathbf{u}}\left(x_{0}, y_{0}\right)\right\|
$$

if normalization (9) is used. As a consequence of Eq. (11) they argued that the leading eigenvalue is sensitive to a local feedback only in the overlapping region of the direct and adjoint global modes. 


\section{Sensitivity to a perturbation of the base flow}

Recently, Marquet et al. ${ }^{13}$ studied the modification $(\delta \sigma)$ of the eigenvalue caused by a structural perturbation acting on the base-flow equations. If we consider an arbitrary variation of the base flow, the eigenvalue drift could be written as

$$
\delta \sigma=\frac{\left(M^{+}, \delta \mathbf{U}_{b}\right)}{\left(\hat{\mathbf{u}}^{+}, \hat{\mathbf{u}}\right)}
$$

where $M^{+}$is

$$
M^{+}=\hat{\mathbf{u}}^{*} \cdot \nabla \hat{\mathbf{u}}^{+}-\nabla \hat{\mathbf{u}}^{*} \cdot \hat{\mathbf{u}}^{+} .
$$

Equation (12) describes the effect of the base-flow variation on each global mode but it does not give hints about how to physically produce relevant base-flow modifications susceptible to modify the stability of a global mode. In the perspective of control, Marquet $\mathrm{et} \mathrm{al.}{ }^{13}$ consider a steady force $(\delta \mathbf{F})$, non-dimensional with the same normalization used for the equations, acting at base flow level,

$$
\begin{aligned}
& \mathbf{U}_{b} \cdot \nabla \mathbf{U}_{b}+\nabla P_{b}-\frac{1}{R e} \nabla^{2} \mathbf{U}_{b}=\delta \mathbf{F}, \\
& \nabla \cdot \mathbf{U}_{b}=0 .
\end{aligned}
$$

They ${ }^{13}$ have shown by a perturbation analysis that

$$
\delta \sigma=\frac{\left(\mathbf{U}_{\mathbf{b}}^{+}, \delta \mathbf{F}\right)}{\left(\hat{\mathbf{u}}^{+}, \hat{\mathbf{u}}\right)} .
$$

In (15), $\mathbf{U}_{\mathbf{b}}{ }^{+}$is the adjoint base-flow velocity, solution of the following problem:

$$
\begin{aligned}
& \nabla \mathbf{U}_{\mathbf{b}} \cdot \mathbf{U}_{b}^{+}-\mathbf{U}_{\mathbf{b}} \cdot \nabla \mathbf{U}_{\mathbf{b}}^{+}-\nabla P_{b}^{+}-\frac{1}{R e} \nabla^{2} \mathbf{U}_{\mathbf{b}}{ }^{+}=M^{+}, \\
& \nabla \cdot \mathbf{U}_{\mathbf{b}}^{+}=0,
\end{aligned}
$$

along with homogeneous boundary conditions at the inlet and on the solid wall and the following condition on the outflow boundary: $P^{+} \mathbf{n}-R e^{-1}\left(\mathbf{n} \cdot \nabla \hat{\mathbf{U}}_{\mathbf{b}}^{+}\right)=-\left(\mathbf{U}_{\mathbf{b}} \cdot \mathbf{n}\right) \mathbf{U}_{\mathbf{b}}^{+}+\left(\hat{\mathbf{u}}^{*} \cdot \mathbf{n}\right) \hat{\mathbf{u}}^{+}$.

\section{Passive control}

We apply now the analysis concisely summarized above to investigate the effect of a small body placed in the flow used as passive control device. In particular, we consider a small cylinder of diameter $d^{*}$ located at the position $\left(x_{0}, y_{0}\right)$. A sketch of the controlled diffuser configuration, in which the cylinder is placed along the centerline, is reported in Fig. 2.

In the context of sensitivity analysis, the presence of the cylinder is modeled only by the force that it exerts on the flow, which is a pure drag force in the present case, without any change of the

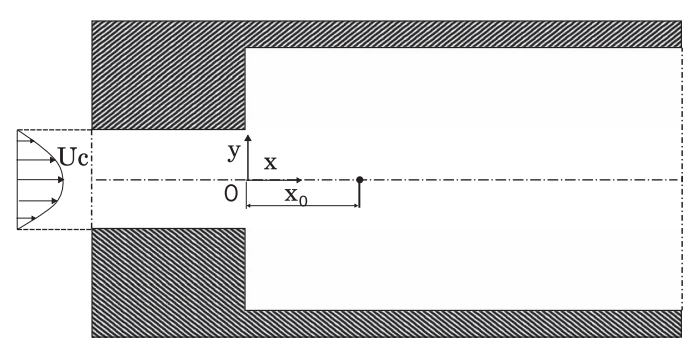

FIG. 2. Channel geometry with the control cylinder. 
domain topology. Indeed, it is well-known that, if the flow Reynolds number based on the cylinder diameter, $R e_{d^{*}}$, is low enough, the circular cylinder experiences a steady drag force.

This force is introduced in the equations as a localized force per unit mass through a Dirac function, so that when this is normalized in agreement with the normalization used for the NavierStokes equations (14), the force $\delta \mathbf{F}$ is given by

$$
\delta \mathbf{F}=-\frac{1}{2} C_{D} d^{*}\|\mathbf{U}(x 0, y 0)\|^{2} \frac{\mathbf{U}(x 0, y 0)}{\|\mathbf{U}(x 0, y 0)\|} \delta\left(x-x_{0}, y-y_{0}\right) .
$$

The drag coefficient $C_{D}\left(R e_{d^{*}}\right)$ can be evaluated from the ESDU database. ${ }^{15}$ In the framework of the linear analysis, we consider the drag force linearized with respect to the perturbation $\mathbf{u}$, given by

$$
\delta \mathbf{F} \approx-\alpha\left[\left\|\mathbf{U}_{\mathbf{b}}\right\|\left(\mathbf{U}_{\mathbf{b}}+\mathbf{u}\right)+\left(\frac{\mathbf{U}_{\mathbf{b}}}{\left\|\mathbf{U}_{\mathbf{b}}\right\|} \cdot \mathbf{u}\right) \mathbf{U}_{\mathbf{b}}\right] \delta\left(x-x_{0}, y-y_{0}\right),
$$

in which the flow is decomposed as in (2) and $\alpha=\frac{1}{2} d^{*} C_{D}\left(R e_{d^{*}}\right)$.

The structural perturbation acts both at the perturbation level by modifying in a direct way the structure of the eigenvalue problem and, indirectly through the perturbation of the base flow by the steady force. The linearized eigenvalue variation caused by the control cylinder can be written, using (7), (15), and (18), as

$$
\delta \sigma=\delta \sigma_{1}+\delta \sigma_{2}=\alpha\left(S_{1}\left(x_{0}, y_{0}\right)+S_{2}\left(x_{0}, y_{0}\right)\right),
$$

where

$$
\begin{aligned}
& S_{1}\left(x_{0}, y_{0}\right)=-\left\|\mathbf{U}_{\mathbf{b}}\right\| \frac{\mathbf{U}_{\mathbf{b}}{ }^{+}\left(x_{0}, y_{0}\right) \cdot \mathbf{U}_{\mathbf{b}}\left(x_{0}, y_{0}\right)}{\left(\hat{\mathbf{u}}^{+}, \hat{\mathbf{u}}\right)}, \\
& S_{2}\left(x_{0}, y_{0}\right)=-\frac{\hat{\mathbf{u}}^{+}\left(x_{0}, y_{0}\right) \cdot\left(\left\|\mathbf{U}_{\mathbf{b}}\left(x_{0}, y_{0}\right)\right\| \hat{\mathbf{u}}\left(x_{0}, y_{0}\right)+\left(\frac{\mathbf{U}_{\mathbf{b}}\left(x_{0}, y_{0}\right)}{\left\|\mathbf{U}_{\mathbf{b}}\left(x_{0}, y_{0}\right)\right\|} \cdot \hat{\mathbf{u}}\left(x_{0}, y_{0}\right)\right)\right)}{\left(\hat{\mathbf{u}}^{+}, \hat{\mathbf{u}}\right)} .
\end{aligned}
$$

We define $S\left(x_{0}, y_{0}\right)=\left(S_{1}\left(x_{0}, y_{0}\right)+S_{2}\left(x_{0}, y_{0}\right)\right)$, which is a complex-valued function giving the eigenvalue variation caused by introduction of an infinitesimal cylinder at location $\left(x_{0}, y_{0}\right)$. Thus, the function $S$ gives a map of the sensitivity of the leading eigenvalue with respect to the control device position.

\section{E. Numerical method}

The two-dimensional unsteady and steady Navier-Stokes equations (1) and (5), their linearized version and the corresponding adjoint equations (6) and (8) are discretized in space by a finite-element method, using P2-P1 Taylor-Hood elements: six-node quadratic triangular elements with quadratic interpolation for velocities (P2) and three-node linear triangular elements for pressure (P1) (see Taylor and Hood ${ }^{16}$ for details). The meshes as well as the discrete matrices resulting from Galerkin finite-element method are generated with the software FREEFEM++ (http://www.freefem.org).

The semidiscrete unsteady equations are advanced in time by a backward differentiation formula of order 2 with a linear second order extrapolation for the convective term (see Quarteroni et al. ${ }^{17}$ ). The steady nonlinear base-flow equations are solved via a Newton-Raphson iterative method and the solution of the related linear systems is carried out via Unsymmetric Multifrontal sparse LU Factorization PACKage (UMFPACK) or MUltifrontal Massively Parallel sparse direct Solver (MUMPS), two sparse direct LU solvers.

Once the problem (6) is discretized in space, we have to solve a generalized eigenvalue problem in the form,

$$
\mathbf{A}\left(R e, \mathbf{U}_{b}\right) \cdot \hat{\mathbf{q}}=\sigma \mathbf{B} \cdot \hat{\mathbf{q}},
$$

where $\hat{\mathbf{q}}$ is a vector collecting all the discrete unknowns of the problem, i.e., velocity components and pressure on each degree of freedom of the discrete problem, B is a matrix which has zeros on the rows corresponding to the boundary conditions and, on the remaining parts, it is equal to a mass matrix. The matrix A contains the discretization of the differential operators and includes 
the boundary conditions. The adjoint eigenvector $\hat{\mathbf{q}}^{+}$is computed as the solution of the following problem:

$$
\mathbf{A}^{H}\left(R e, \mathbf{U}_{b}\right) \cdot \hat{\mathbf{q}}^{+}=\sigma^{*} \mathbf{B}^{H} \cdot \hat{\mathbf{q}}^{+},
$$

where ()$^{H}$ stands for Hermitian. The eigenvalue problems (21) and (22) are solved by using a power method along with a shift-invert strategy, implemented with the libraries PETSc (Portable Extensible Toolkit for Scientific Computation). ${ }^{18}$

\section{RESULTS}

\section{A. Base flow}

A computational domain is considered here having $L_{1}=3 d, L_{2}=50 d$ and, as mentioned in Sec. II, $E R=3$, which is similar to what is done in the literature for the same flow (see, for instance, Hawa and Rusak $^{7}$ and Battaglia et al. ${ }^{5}$ ). A mesh composed of triangular elements is generated though the Delaunay-Voronoi algorithm. Since in our analysis the base flow is the steady symmetric solution, we consider a mesh $M_{b f h}$ of half of the channel, refined near the corner edges, with about $3.7 \times 10^{4}$ nodes and $7.2 \times 10^{4}$ triangles. Grid convergence tests have been carried out to verify the independence of the computed base flow with respect to the spatial discretization. In these tests, not reported here for the sake of brevity, grid convergence is estimated monitoring the position of the reattachment point of the flow on the lateral walls, since it is known to be particularly sensitive to the spatial resolution.

The flow in a channel with ER $=3$ is known to become unstable at a critical Reynolds number $R e_{c} \approx 81$. Nonetheless, the unstable symmetric solution, which represents the base flow for the stability and sensitivity analyses, can be numerically computed by enforcing the symmetry as above explained. As an example, the the unstable symmetric flow computed at $R e=100$ is depicted by streamlines in Fig. 3(a). As can be seen in Fig. 3(b), the size of the recirculating region in this symmetric flow configuration increases almost linearly with $R e$, as well-known from the literature (see, e.g., Refs. 2, 8, and 19). As an example, the data from Alleborn et al. ${ }^{8}$ are also reported in Fig. 3(b); an excellent quantitative agreement is obtained.

\section{B. Stability analysis}

Depending on the initial guess for the Newton method and using the mesh of the entire channel $M_{b f}$, obtained from $M_{b f h}$ with a reflection along the centerline, we could find both asymmetric and symmetric solutions for $R e \geq R e_{c}$. Fig. 4(a) shows the asymmetric stable solution at $R e=100$, depicted by streamlines, for a visual comparison with the unstable symmetric one reported in Fig. 3(a). Figure 4(b) shows the streamwise position of the reattachment points $\left(x_{r}\right)$ of the flow as a function of the Reynolds number. For $R e<R e_{c}$ the reattachment points on the two walls coincide and the same linear behavior as in Fig. 3(b) is reported. For $R e>R e_{c}$, due to the asymmetry

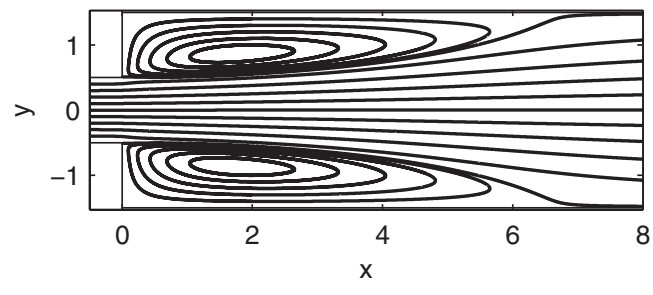

(a)

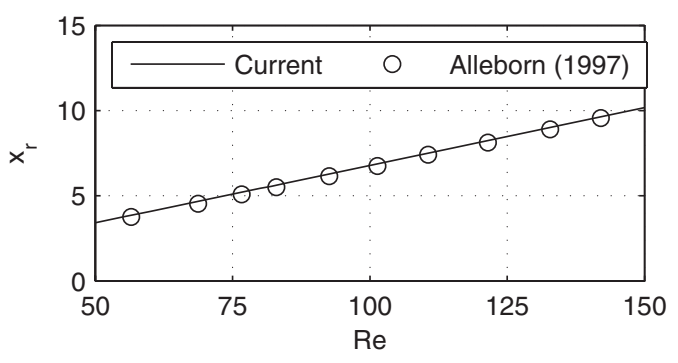

(b)

FIG. 3. (a) Streamlines of the symmetric unstable solution at $R e=100$. (b) Location of the reattachment points as a function of the Reynolds number in the symmetric case. 


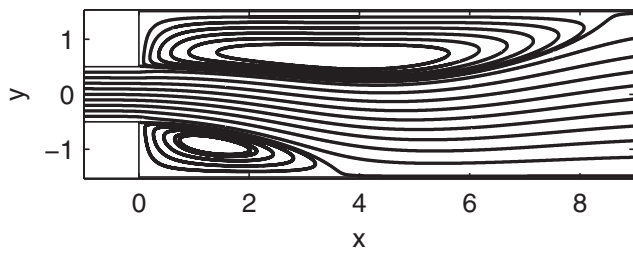

(a)

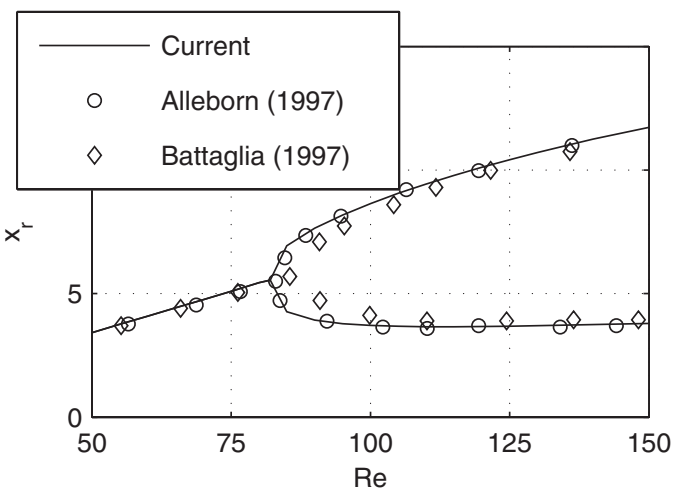

(b)

FIG. 4. (a) Streamlines of the asymmetric stable solution at $R e=100$. (b) Location of the reattachment point as a function of the Reynolds number.

of the flow configuration, the two reattachment points differ as reported in the figure. Data from Alleborn et al. ${ }^{8}$ and Battaglia et al..$^{5}$ are also shown, in order to validate the present results. An overall very good agreement is observed.

The global stability of the base flow is investigated by looking for the leading global mode $(\hat{\mathbf{u}}, \hat{p})$, defined as the global mode of largest growth rate.

The domain for the stability problem is the entire channel, and in order to limit the computational costs, $L_{2}$ (see Fig. 1) has been reduced to $L_{2}=35 d$. Tests have been carried out showing that the results of the stability and sensitivity analysis are not affected by this reduction. This is also confirmed by the results reported in Sec. III C. Once the base flow has been computed on the mesh $M_{b f h}$, it is interpolated on a mesh $M_{s t}$, discretizing the whole diffuser and having practically the same resolution as $M_{b f h}$, on which the stability problems (21) and (22) are solved. The mesh has about 4.1 $\times 10^{4}$ nodes and $9.6 \times 10^{4}$ triangles. The eigenvalue of the leading global mode has been used to check the sensitivity of the results to the grid resolution, justifying the use of this mesh. Figure 5(a) shows the growth rate of the leading global mode as a function of the Reynolds number. As expected, the imaginary part of both the eigenvalue and of the global mode is null, because it is linked to a stationary mode. The growth rate crosses the real axis at the critical Reynolds number $R e_{c}=81.2$, at which the base flow becomes unstable. This value of the critical Reynolds number is in excellent agreement with works of other authors. ${ }^{5,7,11}$

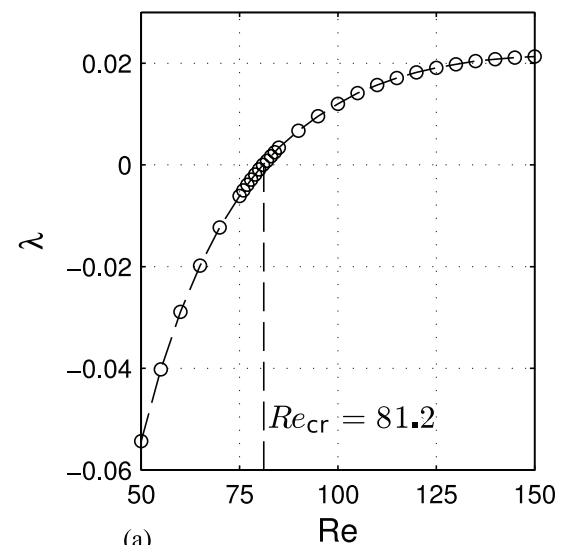

(a)
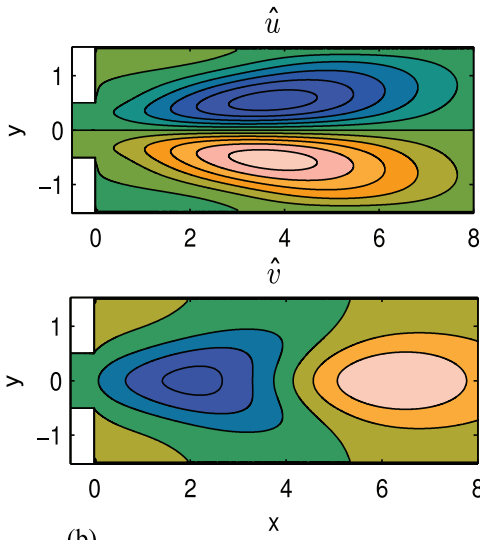

(b)
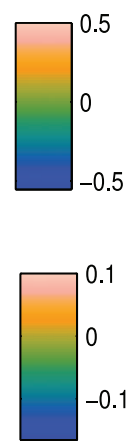

FIG. 5. (a) Growth rate $\lambda$ of the leading global mode as a function of the Reynolds number Re. (b) Leading global mode at $R e=90:$ streamwise $(\hat{u})$ and cross-stream $(\hat{v})$ velocity. 


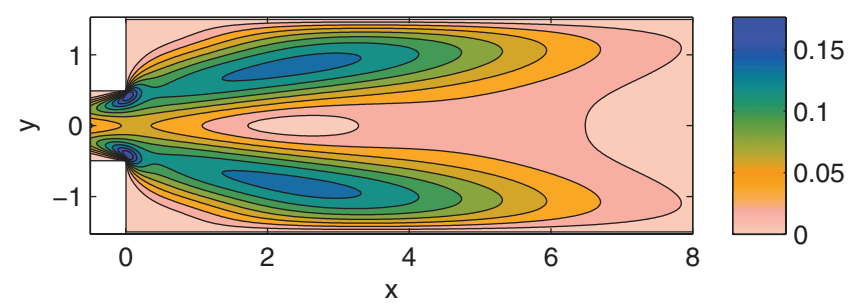

FIG. 6. Modulus of the velocity field $\left(\hat{\mathbf{u}}^{+}\right)$of the adjoint mode at $R e=90$.

The leading global mode at $R e=90$ is depicted in Fig. 5(b) by its velocity fields. The streamwise velocity is antisymmetric with respect to the centerline $y=0$, while the cross-stream velocity is symmetric with values of alternating sign in the streamwise direction. The modulus of the velocity reaches its peak value at $x \approx 4$ and slowly decays in the streamwise direction further downstream. The adjoint eigenvector for the leading global mode is displayed in Fig. 6. The adjoint field represents a sort of Green function for the receptivity of the corresponding global mode. The scalar product of the adjoint eigenmode with any forcing function and/or initial condition indeed provides the amplitude of the associated direct mode. ${ }^{12}$ The region of maximum receptivity for the asymmetric instability is located close to the expansion corners. The case at $R e=90$ shown in the previous figures has been chosen as an example of the behavior for Reynolds numbers moderately larger than the critical value. For small changes in the Reynolds number, the spatial distribution (structure) of the unstable mode and of its adjoint remains qualitatively the same.

\section{Sensitivity to a structural perturbation and base-flow modifications}

Figure 7 shows the overlapping region of the direct and adjoint global modes at the same Reynolds number as in Figs. 5(b) and 6. From Eq. (11) and following Giannetti and Luchini ${ }^{12}$ we can argue that the core of the instability is localized in two symmetric lobes across the separation area. The information from Fig. 7 justifies the choice of a smaller domain for the stability calculation. Indeed, Giannetti and Luchini ${ }^{12}$ have shown that the eigenvalue evaluation is accurate provided that the computational domain includes its instability core.

The sensitivity field $M^{+}$(13) to a perturbation in the base flow (Sec. II C) is generally a twodimensional complex vector. However, we deal here with a stationary mode, thus both the eigenvalue and eigenvectors are real. Consequently, relations (12) and (13) show that, no matter the base-flow modification, the mode keeps being stationary since $\delta \omega=0$, and (12) can be used to compute directly the growth rate variation. The sensitivity $M^{+}$is plotted in Fig. 8. It is a two-dimensional real vector field represented both by streamlines, giving the local orientation of the sensitivity field, and by colours indicating its magnitude. The largest magnitudes are reached inside the recirculation region, similarly to what previously observed for the instability core, but also near the expansion corners, due to the singularity of the geometry. Therefore, if we consider an arbitrary base-flow modification $\delta \mathbf{U}_{b}$ that, anywhere in the flow, is oriented in the same direction as the growth rate sensitivity, the variation of the growth rate is positive $(\delta \lambda>0)$ which indicates a destabilization of the flow.

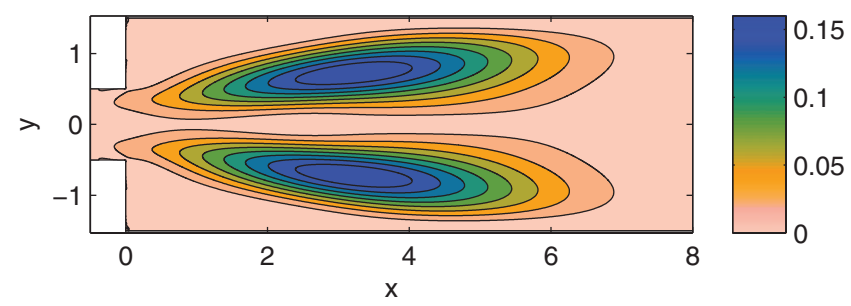

FIG. 7. Structural sensitivity with respect to perturbation at $R e=90$. 


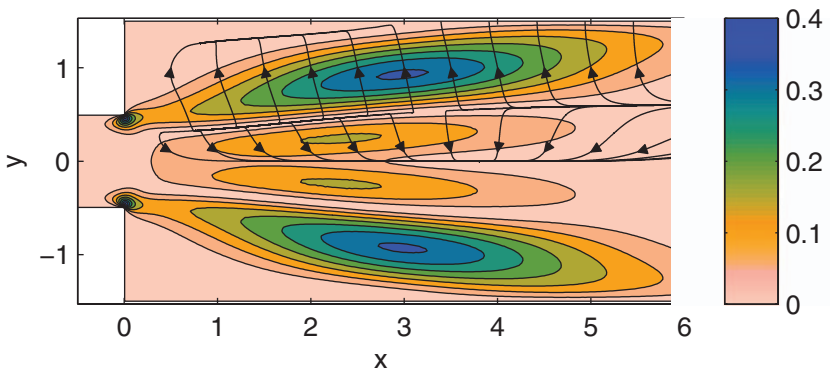

FIG. 8. Sensitivity with respect to base-flow modification at $R e=90$.

We now consider the sensitivity with respect to a steady force in the momentum equation (5). As mentioned before $M^{+}$is real, thus the adjoint base flow, solution of problem (16) is real too. Relation (15) shows that $\delta \omega=0$, then the forced global mode keep being stationary. The adjoint base-flow velocity is plotted in Fig. 9. The field is symmetric and we can identify the local maximum of the growth rate sensitivity to a steady force. The most sensitive area is around the expansion corners. A weaker local maximum is located close to the centerline, near the expansion. A local steady force oriented in the opposite direction to the arrows plotted in Fig. 9 induces a negative variation of the growth rate $(\delta \lambda<0)$ which is proportional to the local magnitude of the sensitivity function.

\section{Application to passive control}

The passive control strategy presented in Sec. II D is applied here. In our case $S_{1}$ and $S_{2}$ are real and we obtain that

$$
\delta \lambda\left(x_{0}, y_{0}\right)=\alpha S\left(x_{0}, y_{0}\right)
$$

The sensitivity fields $S_{1}, S_{2}$ (see Eqs. (20a) and (20b)) and $S=S_{1}+S_{2}$ are plotted in Fig. 10, where a negative value indicates a stabilizing effect. First, we see that the two contributions are of the same order of magnitude, thus none of these two components can be neglected. The map shown in Fig. 10(c) indicates that there is a symmetric region near the expansion section where the introduction of the considered forcing is expected to yield a substantial decrease of the unperturbed growth rate.

Figure 10(c) also shows that the largest stabilizing effect is obtained if the control is located in a point on the centerline, close to the expansion section. The maps in Fig. 10 have been obtained at $R e=90$; the sensitivity maps for moderately different Reynolds numbers are qualitatively similar. To better quantify the effects of Re, Fig. 11 shows $S$ as a function of the location of the control force on the centerline, obtained at different Reynolds numbers. As the Reynolds number is increased, the optimal position moves slightly downstream and the sensitivity value decreases, thus it is necessary to rise the force amplitude to obtain the same eigenvalue drift (see Eq. (23)).

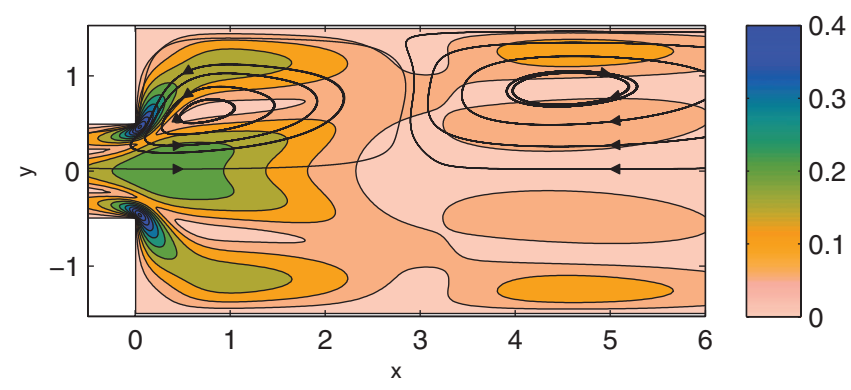

FIG. 9. Sensitivity with respect to steady forcing at $R e=90$. 

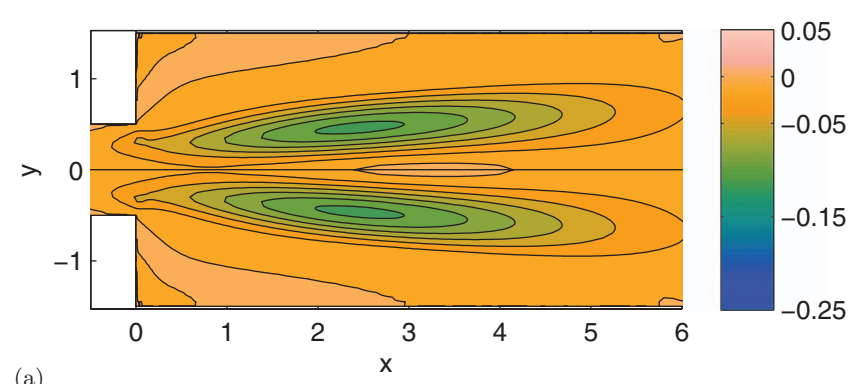

(a)
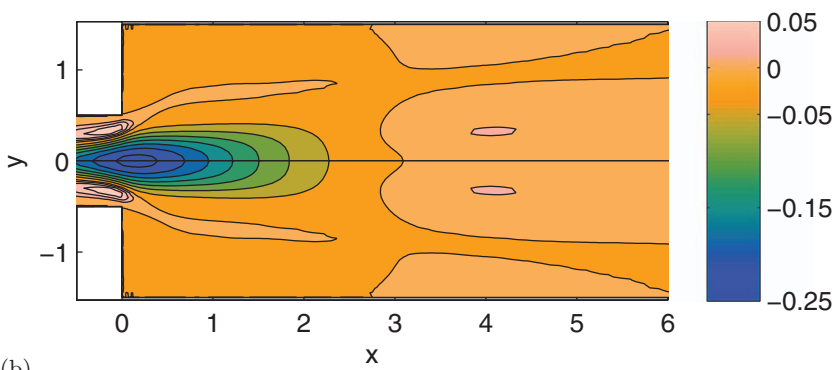

(b)

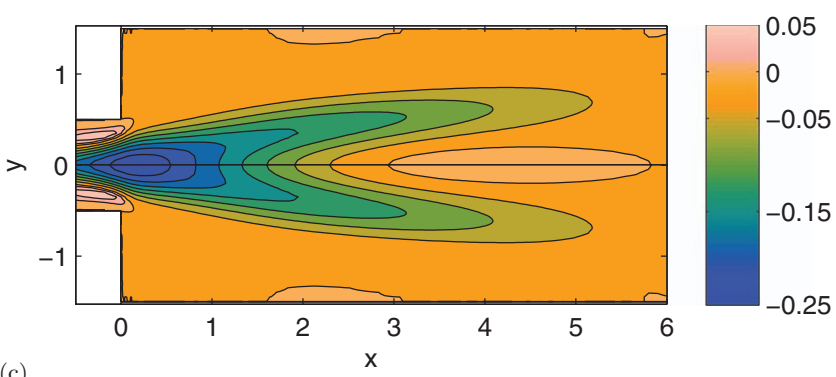

(c)

FIG. 10. $S_{1}\left(x_{0}, y_{0}\right)$ field (a), $S_{2}\left(x_{0}, y_{0}\right)$ field (b), and their sum $S\left(x_{0}, y_{0}\right)$ (c) at $R e=90$.

For each value $\alpha$, a linear estimation of the growth rate of the global mode may be obtained using the described sensitivity analysis. The growth rate of the forced flow could be evaluated, using (19) as

$$
\bar{\lambda}(R e, \alpha)=\lambda_{0}(R e)+\alpha S\left(x_{0}, y_{0}\right),
$$

where $\lambda_{0}$ is the unforced growth rate. On the basis of this analysis, and considering that the forcing amplitude is related to the diameter of the control cylinder by Eq. (17), it is possible to estimate

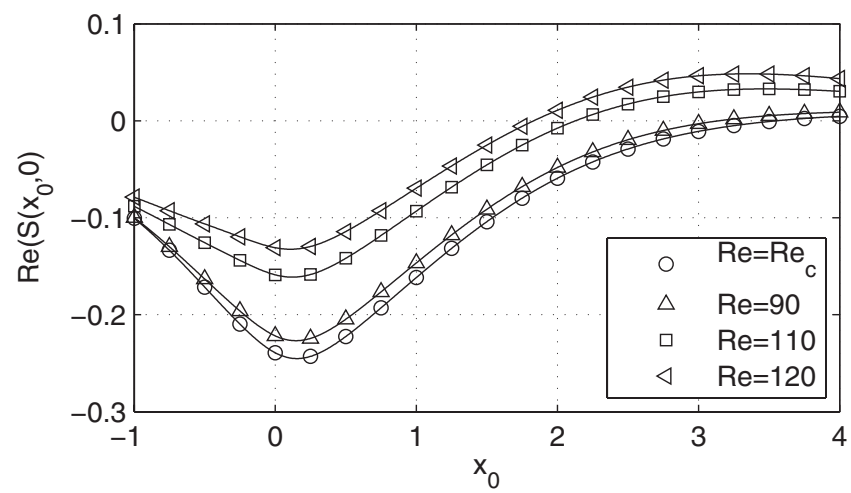

FIG. 11. $S\left(x_{0}, 0\right)$ as a function of the control location along the centerline, $x_{0}$, at various Reynolds numbers. 
TABLE I. Computed values of $\alpha$ and related amplification factor $\lambda$ for three different diameters of the control cylinder located in the point $(0.15,0)$.

\begin{tabular}{|c|c|c|c|c|c|c|}
\hline & \multicolumn{2}{|c|}{$d^{*}=0.01$} & \multicolumn{2}{|c|}{$d^{*}=0.02$} & \multicolumn{2}{|c|}{$d^{*}=0.05$} \\
\hline & $\alpha$ & $\lambda$ & $\alpha$ & $\lambda$ & $\alpha$ & $\lambda$ \\
\hline$R e=90$ & 0.06 & -0.069 & 0.076 & -0.01 & 0.109 & -0.018 \\
\hline$R e=100$ & 0.056 & 0.002 & 0.07 & -0.001 & 0.103 & -0.008 \\
\hline$R e=110$ & 0.052 & 0.008 & 0.066 & 0.005 & 0.098 & 0.0003 \\
\hline$R e=120$ & 0.049 & 0.012 & 0.063 & 0.01 & 0.093 & 0.006 \\
\hline
\end{tabular}

the cylinder diameter and position in order to suppress the instability. For instance, Table I shows the values of $\alpha$ computed for three different diameters of the control cylinder $\left(d^{*}=0.01,0.02\right.$, and 0.05 ) located in the point $(0.15,0)$, which is almost coincident with the point of maximum control sensitivity, and the corresponding values of the amplification factor $\lambda$, at different values of the Reynolds number.

Finally, we have repeated the linear stability analysis for the case in which a control cylinder identified by the procedure described above is effectively placed in the flow field. For the computation of the base flow we used a mesh $M_{c y l}$ with a refinement close to the cylinder position. We obtained the meshes $M_{c y l}^{0.01}, M_{c y l}^{0.02}$, and $M_{c y l}^{0.05}$ from the mesh $M_{c y l}$ by a boolean subtraction operation of the nodes inside cylinder of diameters equal to $0.01,0.02$, and 0.05 , respectively, and by imposing a no-slip boundary condition on the cylinder surface.

As an example, the results of the linear stability analysis carried out for the case with a cylinder having diameter $d^{*}=0.05$ and symmetrically placed on the centerline at different positions of its center $\left(x_{0}\right)$ are shown in Fig. 12.

If we consider the cylinder placed at position $(0.15,0)$, as it is the case of Table I, Fig. 12 shows that the critical Reynolds number is delayed to $R e_{c r} \simeq 116$. This result is in good agreement with the data of Table I, which predict that the cylinder, which corresponds to the value of alpha indicated by the triangular symbol, is able to stabilize the flow up to $R e_{c r} \simeq 110$. We remind that the proposed passive control is based on several approximations: the flow equations are linearized around the uncontrolled flow, the cylinder is modeled as a pointwise drag force, and the analysis is valid for infinitesimal drag forces. The effect of all the approximations mentioned above, for instance, can explain the fact that the position corresponding to the maximum $\operatorname{Re}_{c}$ in Fig. $12(x \simeq 0.3)$ does not coincide with the position of the minimum of the sensitivity function $S(x \simeq 0.15)$. The neglected nonlinear effects are discussed in more details in Sec. III E.

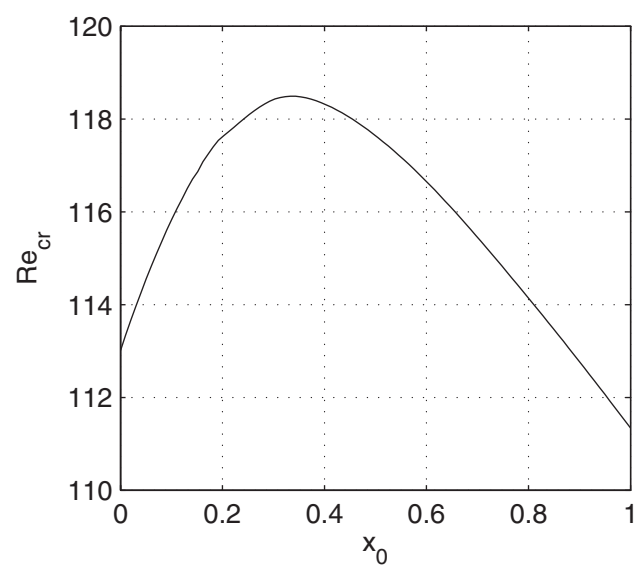

FIG. 12. Critical Reynolds number $R e_{c}$ as a function of the $x_{0}$ position of the cylinder center. The cylinder has a diameter equal to 0.05 and it is placed on the centerline. 




FIG. 13. Power extracted from the flow by the force whose magnitude and direction is the ones needed for a given shift of the amplification factor.

In order to provide a physical explanation for the stabilizing effect of the proposed control, an energetic analysis of the flow can be carried out, with focus on the power introduced/extracted by the controlling force. In this respect, it has been shown that the introduction of a localized control force, which is subsequently exploited by a physical device as the cylinder in our case, affects the unstable eigenvalue as illustrated in Fig. 9. In particular, the sensitivity reported in Fig. 9 is a vector field identifying, for a given shift of the amplification factor and for each point in space, the needed localized control force. From an energetic view point, by a scalar multiplication between this sensitivity vector field and the velocity field of the base flow, with the proper sign of the force in order to lead to a stabilizing effect, a scalar field can be obtained representing, for each point, the power that the control force must introduce/extract from the flow for a given shift of the amplification factor. This map, reported in Fig. 13 for the case of $R e=100$, shows that the control force, in the high sensitivity region, always acts as a damper, i.e., it extracts energy from the base flow. The need for an additional extraction of energy is reasonable, thinking that in this particular flow an increase of the Reynolds number, which leads to a destabilization of the symmetric configuration, is associated to a decrease of energy dissipation.

\section{E. Discussion on the validity of the linearized maps}

The passive control described in this paper has been designed on the basis of Eq. (24), in which the map $S(x, y)$ is evaluated by a linearized analysis carried out around the uncontrolled flow. The sensitivity analysis is fundamentally linear since it is based on the evaluation of a gradient. Therefore, Eq. (19) remains accurate only for small values of the force amplitude $\alpha$. To asses how a finite force amplitude alters the results of the sensitivity analysis we consider a force of increasing amplitude $\alpha$, given by Eq. (18) and placed at the location $(0.15,0)$, and we evaluate the growth rate $\lambda_{f}$ of the forced flow directly taking into account the drag force (17) both in the base-flow equations and in the linearized perturbation problem. The forced base flow is thus obtained by solving the nonlinear problem (14) in which the forcing is given by the part of $F$ (Eq. (18)) depending only on the base flow. Moreover, the growth rate is evaluated by solving the stability problem (6) by using the computed forced base flow and by considering the part of $F$ proportional to the perturbation. The force in the continuous formulation is proportional to a localized Dirac function. In the discretized system, the Dirac function is regularized by substituting it with a normalized gaussian function of width 0.01 centered at $\left(x_{0}, y_{0}\right)$. Another strategy has been also tested, in which the Dirac function applied on a node of the discretization has been replaced by the Lagrangian basis function associated to the same node and to the finite-element grid, properly normalized. The differences between the two approaches described above were definitely negligible. 


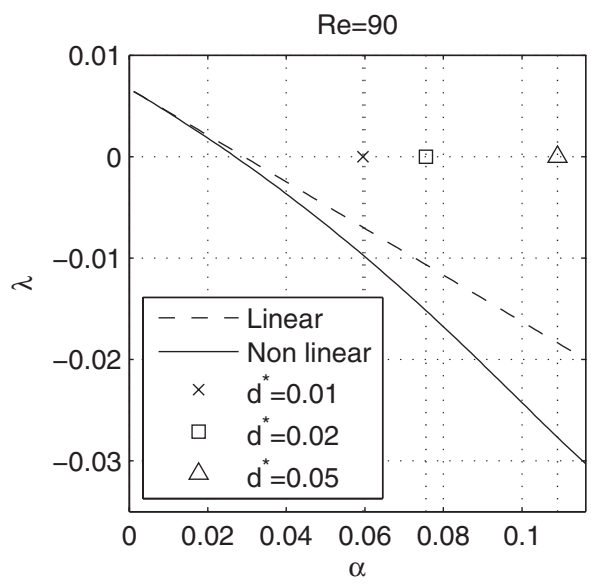

(a)

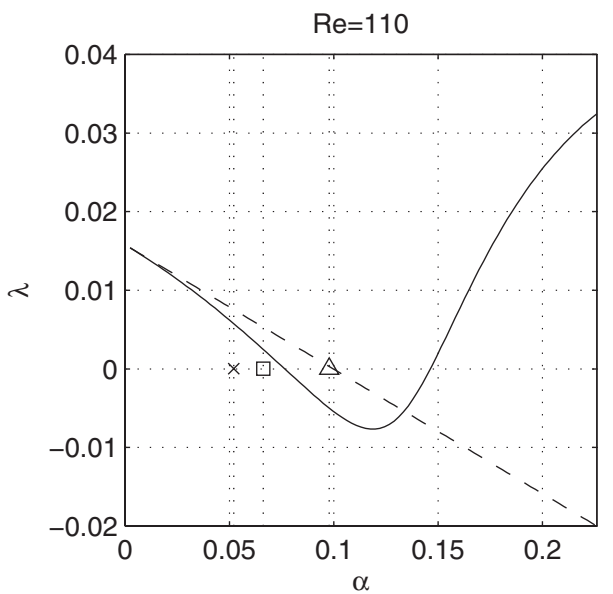

(c)

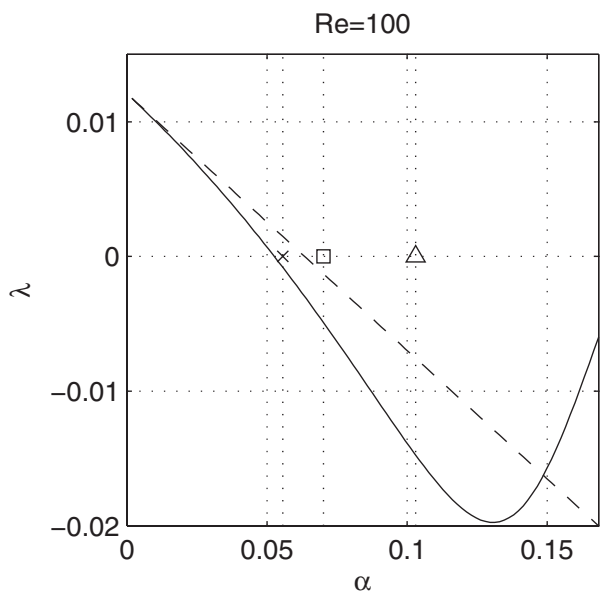

(b)

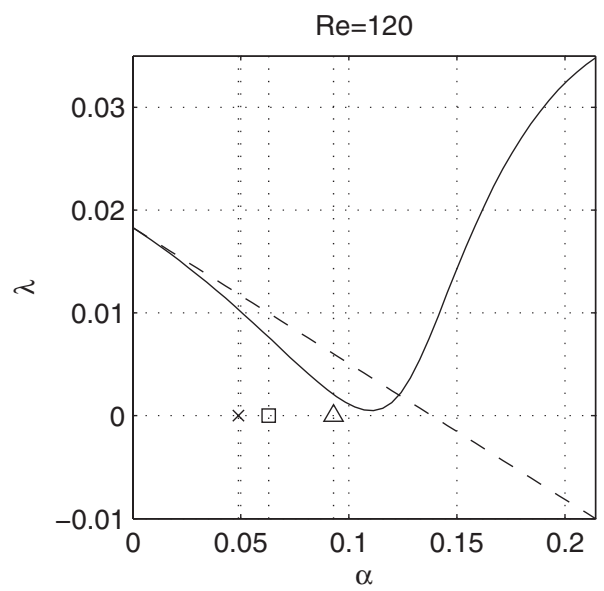

(d)

FIG. 14. (a)-(d) Linear estimation $\bar{\lambda}$ (dashed line denoted as "linear") and $\lambda_{f}$ (continuous denoted as "nonlinear") for various Reynolds numbers, as a function of force amplitude $\alpha$.

Results are shown in Fig. 14, where $\bar{\lambda}$ (Eq. (24)) is evaluated for increasing values of the force $(\alpha)$, and compared with the corresponding value of $\lambda_{f}$. The cross, square, and triangle symbols in the figure indicate the force amplitudes equivalent to control cylinders with diameter equal to 0.01 , 0.02 , and 0.05 , respectively, according to Eq. (17). For small amplitudes of the force $(\alpha \ll 1) \lambda_{f}$ and $\bar{\lambda}$ coincides to the plot accuracy. This result validates the sensitivity analysis and, in particular, the accuracy of the gradient estimation through the sensitivity function. For larger amplitudes nonlinear effects become important and, in particular, for $R e \geq 100$, we observe the presence of a local minimum for $\lambda_{f}$ at $\alpha=\alpha_{c}$. For $\alpha<\alpha_{c}$ we have that $\lambda_{f}<\bar{\lambda}$ indicating that in this particular case the nonlinear effects increase the stabilizing action of the control force. However, if the growth rate at the local minimum remains positive, it is not possible to control the instability for any value of $\alpha$ in the considered range, despite the indications from the sensitivity analysis.

A similar case is described in Camarri and Iollo ${ }^{14}$ for the control of the vortex-shedding in the wake of a confined cylinder, showing that the indications of the linear analysis carried out around the uncontrolled flow may give indications for flow stabilization that quickly loose accuracy as the actuation intensity increases, due to nonlinear effects, especially as the flow Reynolds number is increased. To overcome this problem, in Camarri and Iollo ${ }^{14}$ it is proposed to design the control iteratively, i.e., to use the results of a linearized analysis in its validity range and to progress in the 


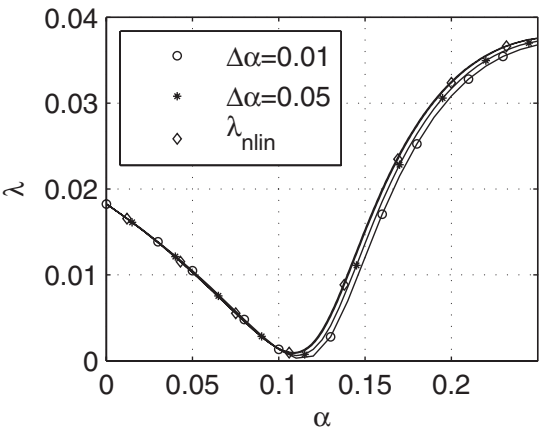

(a)

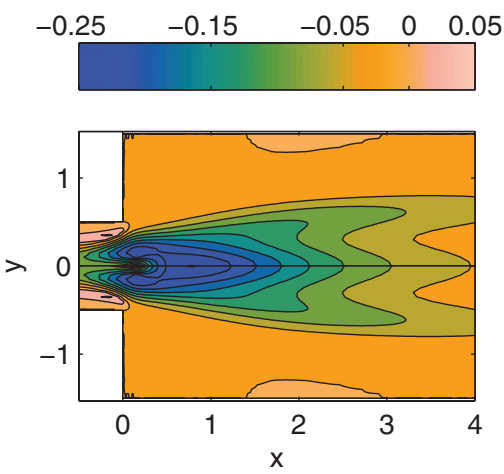

(b)

FIG. 15. Eigenvalue growth rate as a function of $\alpha$ at $R e=120$. Nonlinear estimation compared with that computed by the sensitivity analysis iteratively repeated each $\Delta \alpha$ (a). Sensitivity map for the controlled flow at $\alpha=\alpha_{c}$ (b).

control design by linearizing the equations again around the partially controlled flow. This strategy can give the position of the actuators as an output of the control design, as well as its intensity. A necessary step for the application of this strategy is to estimate the range of validity of the linearized analysis for the considered flow Reynolds number. An example in this direction is provided in Fig. 15(a), where we compare the curve $\lambda_{f}(\alpha)$ with the approximate one computed by repeating the sensitivity analysis for each $\Delta \alpha$; the Reynolds number is 120 and two different values of $\Delta \alpha$ have been used. The figure shows that, for both values of $\Delta \alpha$ the reference curve is well recovered by the approximate analysis for the considered range of $\alpha$, thus giving an indication for the range of confidence $\Delta \alpha$ of the linearized analysis.

When $\alpha$ is increased, the nonlinear effects progressively affect also the sensitivity maps on which the control is based, changing their shape with respect to those obtained considering the uncontrolled flow. As an example, the sensitivity map for the controlled flow at the value of $\alpha$ corresponding to the minimum of the growth rate is depicted in Fig. 15(b). The comparison with the corresponding map for the uncontrolled flow, Fig. 10(c), clearly puts in evidence marked differences near the point where the force acts, indicating that a progressively reduced control margin can be obtained by further increasing the forcing amplitude without changing its position. In particular, when the minimum value for $\lambda$ is obtained, the map should accordingly indicate a null value on the force position, since the map indicates the derivative of $\lambda$ with respect to $\alpha$. The iterative design of the control can take advantage also of the use of linearized sensitivity maps obtained on the partially controlled flow, for example, to change the position of the control cylinder or to introduce a second control cylinder in the flow in appropriate positions.

\section{F. Verification and analysis of the control by DNS}

It is important to underline that the passive control proposed here is based on a linearized analysis carried out around the symmetric state of the flow, which is also the target flow. In this context, stabilizing this flow configuration means that it becomes linearly stable, but information on the attraction basin of this solution cannot be extracted by this analysis, since nonlinearities need to be taken into account to this purpose. In this respect, in order to a posteriori check the robustness of the present control strategy we performed a set of unsteady nonlinear simulations of the flow with the control cylinder. We report here, for example, the results for the case with the cylinder of diameter equal to $d^{*}=0.02$ with its center at position $x_{0}=0.15, y_{0}=0$. The cylinder is inserted impulsively in the simulation once the asymmetric instability is nonlinearly saturated, i.e., when the steady stable asymmetric flow configuration is reached. Note that this is a flow condition that is very different with respect to the target of the control, i.e., the symmetric flow configuration and, therefore, this is a severe test for the proposed control strategy. We ran evolutive simulations at three different values of the Reynolds number, viz., $R e=90,100$, and 110 . In the first two cases, the 


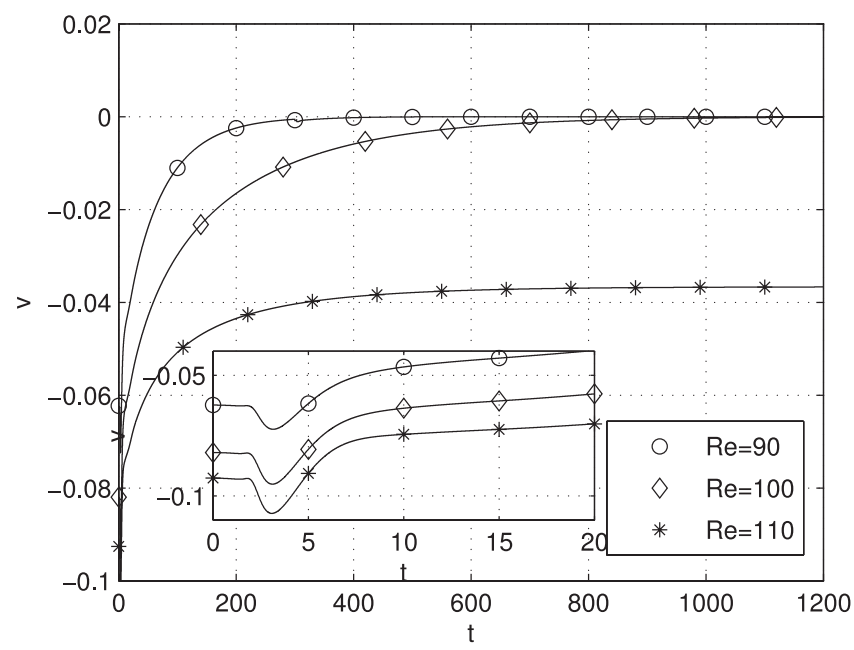

FIG. 16. Time trace of the transverse velocity component at a given point along the channel centerline $(\mathrm{v}=0$ symmetric solution). The box shows the initial transient from the free flow to the controlled one.

sensitivity analysis indicates a negative growth rate, i.e., the suppression of the asymmetric mode. The time trace of the vertical velocity at a given point along the centerline, shown in Fig. 16, reveals that in both cases the asymmetric mode is damped (in that figure the velocity of the uncontrolled case is the one at time $t=0$ ). Note that for the lowest Reynolds number the transient to the symmetric state is shorter than that for the higher Re. At $R e=110$ the stabilizing effect of the cylinder is not enough to suppress the instability, but the flow is less asymmetric than the initial one. Indeed, the values of the vertical velocity component on the symmetry axis have been found to be systematically lower in the controlled case than in the uncontrolled one, as shown, for example, in Fig. 16 for a particular point. Obviously, the described test does not characterize the attraction basin of the stabilized configuration, but it gives an idea of the robustness of the control for a case in which we start controlling when the flow is far from the target flow configuration.

Direct numerical simulation can also be used to investigate the physical mechanisms through which the control acts on the flow. To this aim, we now describe in detail the flow obtained by DNS at $R e=100$, which is one of the three cases reported in Fig. 16. We also describe the transient flow which is obtained at $R e=100$ when the uncontrolled flow is simulated starting from the symmetric unstable solution of the equations, thus showing the growth and saturation of the instability before introducing the control cylinder. Four instantaneous flow snasphots, taken at different time intervals $\Delta T$ measured from the beginning of the simulation, are reported in Fig. 17 by plotting both the streamlines and the vorticity field. As shown, the flow departs from the initial symmetric solution and goes toward one of the two possible stable asymmetric solutions. Note, in particular, how the progressive change in size of the two recirculation regions, and the consequent modification of the vorticity there contained, corresponds to a modification of the vorticity produced at the upper and lower walls of the largest channel. The time needed for this transient depends on the amplitude of the initial perturbations. In this case no perturbation has been explicitly superposed, letting the numerical errors being the source of perturbation. It is not shown here, for the sake of brevity, that the instability starts with an exponential amplification of the unstable mode, which is exactly equal to the one predicted by the linear stability analysis, showing that in this case non-normal transient growth is absent. At the end of the described transient, the flow remains on a stable asymmetric solution.

At this point, the control cylinder identified in Sec. III D, i.e., a cylinder with diameter $d^{*}=0.02$, is impulsively introduced in the DNS simulation at $x_{0}=0.15$. In order to have a precise time reference, we arbitrarily choose the time $t=0$ as the instant at which the cylinder is introduced, as already done in Fig. 16. 

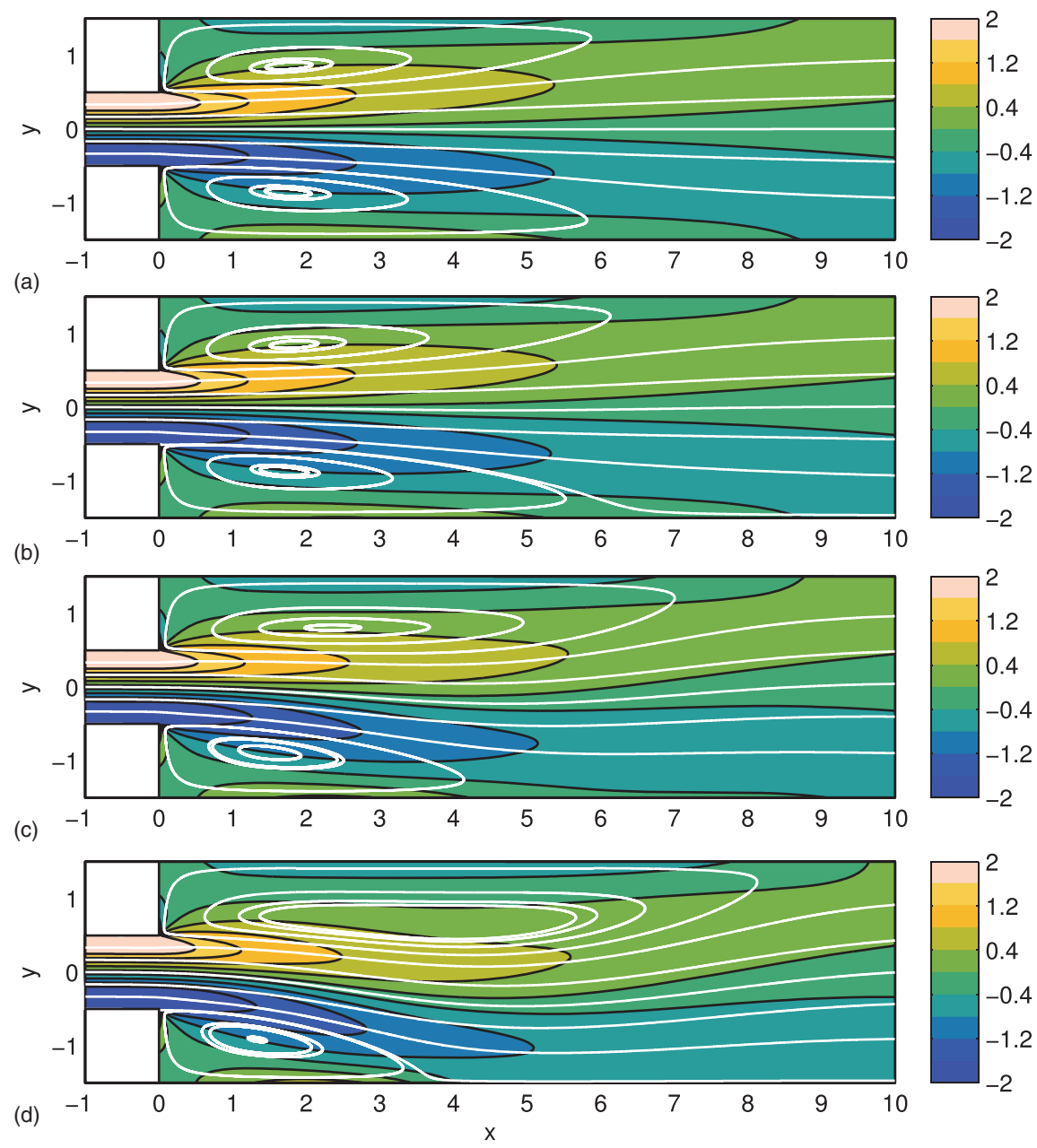

FIG. 17. (a)-(d) Instantaneous flow snapshot of the uncontrolled case at $R e=100$. The growth of the instability is depicted by both the streamlines and the vorticity field. The time interval between each snapshots is $\Delta t=100$.

Some subsequent instantaneous snapshots of the transient flow arising from the introduction of the control cylinder are reported in Fig. 18, showing again the streamlines and the vorticity field. The snapshots are not uniformly distributed in time because the initial transient is dominated by convection while the later stages of the transient flow are dominated by diffusion, so that the characteristic evolutive times are quite different. The sequence of snapshots shows that the symmetric solution is recovered by the control, and this is practically obtained after a time approximately equal to $t=800$. The formation of the wake behind the cylinder is clearly visible in those figures, giving an idea of the convection velocity in central part of the channel and showing that vortex shedding from the cylinder is, as expected, absent. As shown in the figures, the interaction between the vorticity in the wake of the cylinder and that already present in the channel, and continuously generated at the channel walls, is weak. Indeed, the wake of the cylinder is generated at the channel centerline, where the incoming vorticity is very low, and convection is mainly directed along the channel axis. Thus, the convection of the vorticity generated from the cylinder towards the separated regions and the wall is practically negligible. Consequently, except for diffusion, the wake vorticity has a poor interaction with the one already existing in the channel, so that we can exclude that vorticity interaction/cancellation plays a significant role in the instability suppression. Conversely, it is clear by the figures that the main effect of the cylinder, due to the drag force exerted on the flow, is to deviate the streamlines entering the larger channel from the smaller one. In particular, the deviation is asymmetric due to the asymmetry of the flow, and the streamline of the centerline in the smaller 


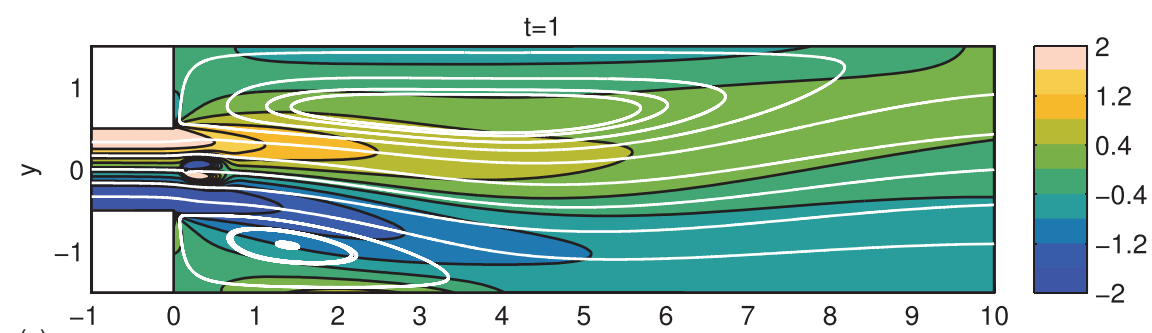

(a)

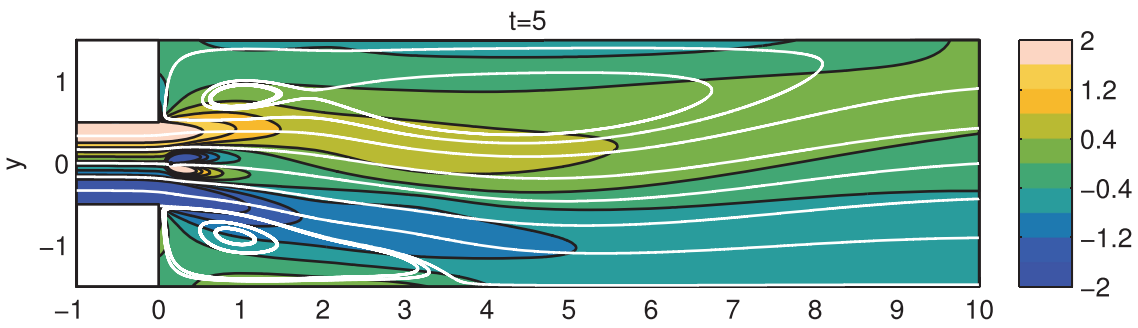

(b)

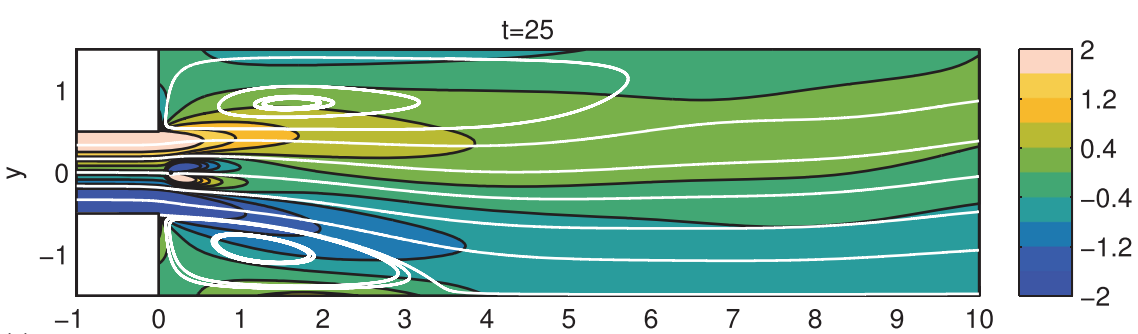

(c)



FIG. 18. (a)-(d) Instantaneous flow snapshot for the controlled case at $R e=100$. The flow is depicted by both the streamlines and the vorticity field.

channel is deviated on the part where separation is smaller. This flow deviation has two main effects in terms of vorticity evolution. First of all, the flow coming from the smaller channel transport a significant amount of vorticity, which is, on both sides, of the same sign as the one contained in the recirculation region. Second, the different pattern of the streamlines leads to a different generation of new vorticity at the walls of the channel, which is of opposite sign with respect to the one contained in the separated regions. Generation of new vorticity, transport of incoming vorticity, and consequent streamlines pattern are in equilibrium in absence of control so that the asymmetric state is stable. The presence of the cylinder breaks this equilibrium in the two ways described above. Although a quantitative analysis of the relative role of the two mentioned effects of the control is difficult, we can try to isolate one of the two effects by performing ad hoc simulations. In particular, we have artificially altered the production of vorticity at the walls of the larger channel in the following way. We consider the evolution of a disturbance superposed to the final symmetric solution, obtained with the control cylinder and shown in Fig. 18(d) (hereafter denoted with the label FSYMCONTR), by properly rewriting the Navier-Stokes equations in terms of the disturbance. If no-slip boundary conditions were imposed on the channel walls for the disturbance, then the same solution as the one 
described in Fig. 18 would be obtained, in terms of disturbance evolution. Conversely, in the present test we impose slip boundary conditions for the disturbance on all walls except those of the smaller channel. In this way a perturbation of the flow with respect to the steady solution FSYMCONTR does not lead to additional vorticity production from the slip walls, thus suppressing one of the two effects of the control identified above. At the same time, FSYMCONTR remains a possible steady configuration of the so-modified flow. Thus, if FSYMCONTR remained stable, this would mean that the convection of the already existing vorticity in the small channel, altered by the cylinder, is by itself sufficient to suppress the instability, and thus it plays a dominant role. If not, this test would show that the production of new vorticity caused by the altered pattern of streamlines is an essential feature in the stabilization of the flow. The DNS simulation, identical to the one documented in Fig. 18 except for the slip conditions applied on the disturbance, shows that the control cylinder is not sufficient to stabilize the symmetric solution, as can be seen by the streamlines and vorticity evolution reported in Fig. 19. As shown in the figure, the missed additional vorticity production induced by the flow perturbation drives the flow towards an asymmetric state which is different from the starting one for the presence of the cylinder and for the alteration of boundary conditions.

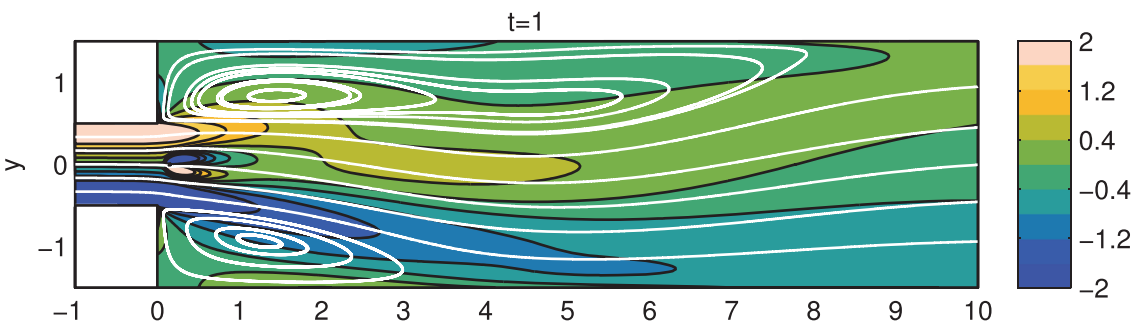

(a)

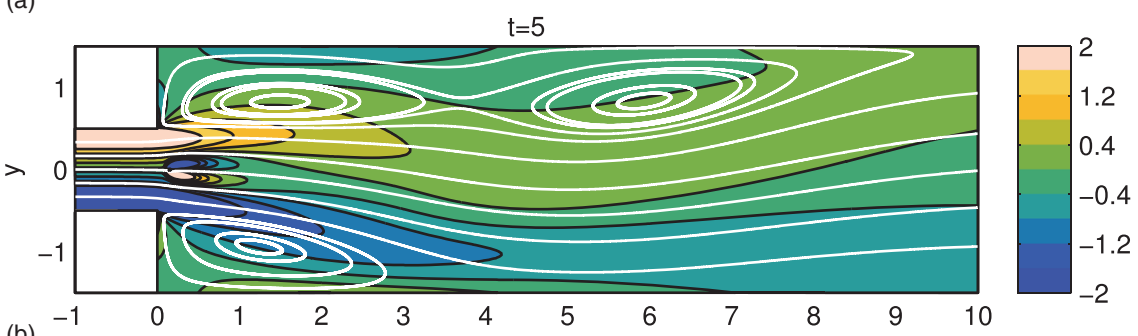

(b)

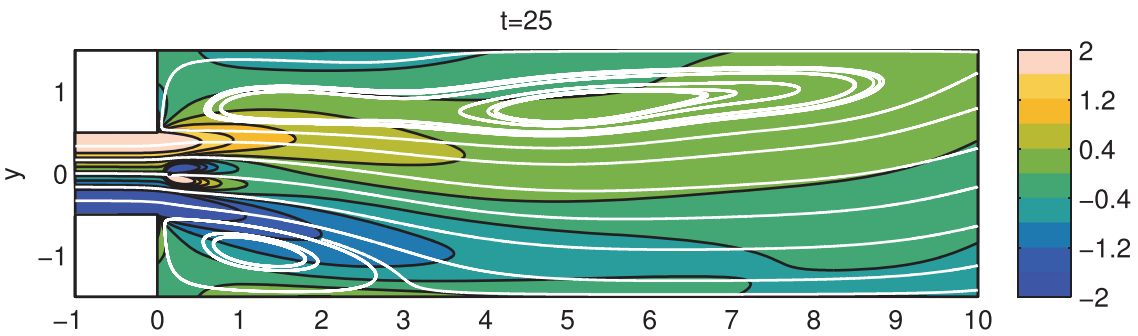

(c)
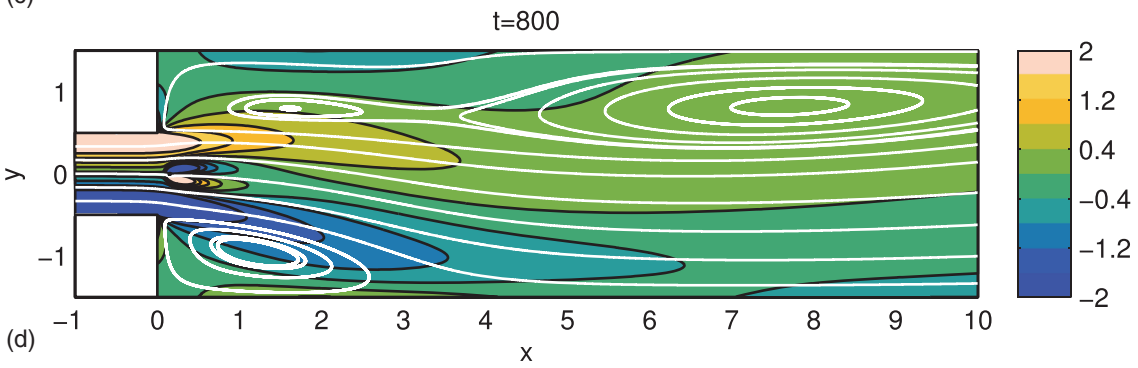

FIG. 19. (a)-(d) Instantaneous flow snapshot for the artificial controlled case, where the production of vorticity at the walls of the larger channel is altered, at $R e=100$. The flow is depicted by both the streamlines and the vorticity field. 

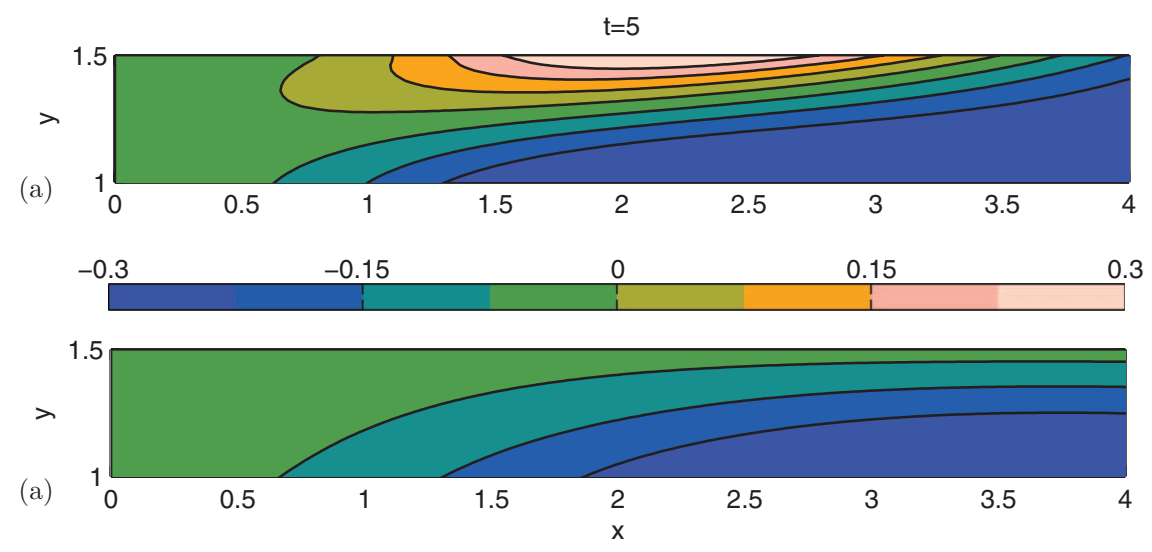

FIG. 20. Zoom of the iso-contours of the vorticity related to the perturbation velocity field. (a) Real controlled case. (b) Artificial controlled case.

The influence of the artificial free-slip conditions imposed on the velocity perturbation can be better appreciated in Fig. 20, where a zoom of the iso-contours of the vorticity associated to this perturbation field is shown near the upper corner of the larger channel, for both the real and the artificial controlled cases (only one instant during the transient is reported for the sake of brevity). It is evident how in the artificial case the production of vorticity associated to the perturbation velocity field is annihilated by the imposed free-slip boundary conditions; conversely, it is quite large in the real controlled flow and this, once again, indicates the importance of this mechanism in the real transient towards the controlled symmetric solution.

For large ER values, Revuelta ${ }^{20}$ stated that the structure of the flow is governed by the momentum flux of the flow entering into the expansion. Although the present ER is much lower than the cases considered by Revuelta ${ }^{20}$ the success of the control strategy might be also explained by the decrease of the momentum flux due to the cylinder drag force, with a consequent decrease of the characteristic Reynolds number based on the momentum flux $J\left(R e^{J}\right)$. In order to check this issue, we computed the critical value of $R e^{J}$ at which the uncontrolled symmetric solution becomes unstable. We found out that in the previously described controlled cases the momentum flux and, consequently, $R e^{J}$, are indeed slightly decreased. However, $R e^{J}$ remains significantly larger than the critical value at which the symmetric solution becomes unstable. Therefore, we can conclude that the success of the control cannot be explained by the above outlined mechanism probably because it holds, as also stated in Revuelta, ${ }^{20}$ only for much larger expansion ratios.

The introduction of the control cylinder yields additional pressure losses through the channel. To quantify this effect, we computed the integral of the dissipation function $\Phi$ in the computational domain (see Fig. 1) for the controlled and uncontrolled cases at $R e=100$. For a steady incompressible flow, as the one considered here, since the contribution of viscous normal stresses at inflow and outflow is negligible, it can be shown from the kinetic energy balance that the integral of the dissipation function is related to the flux of total pressure $P_{t}=P+1 / 2 U^{2}$ as follows:

$$
\int_{\Omega} \Phi d \Omega=-\int_{\partial \Omega} P_{t} \mathbf{U} \cdot \mathbf{n} d \Gamma
$$

We found out that the introduction of the cylinder in the controlled configuration leads to an integral dissipation $6.9 \%$ larger than in the uncontrolled case.

As already stated in the Introduction, attention has been dedicated in the literature also to investigate the effect of a small asymmetry in the channel geometry on the flow stability. ${ }^{9}$ Therefore, an additional test has been carried out here by DNS aimed at checking the capabilities of the proposed control in the presence of the maximum asymmetry among those investigated by Hawa and Rusak. ${ }^{9}$ In particular, the height of the larger channel of the diffuser has been increased only in the upper part by an amount $\delta h_{s}=0.05$. The position and diameter of the cylinder are those identified for the symmetric case $\left(x_{0}=0.15\right.$, with the cylinder center is on the symmetry line of 


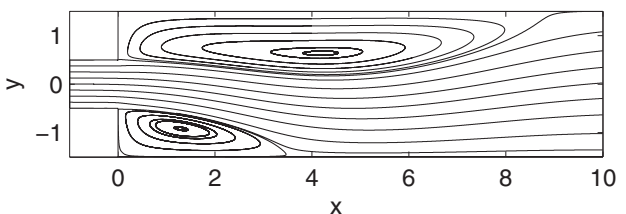

(a)

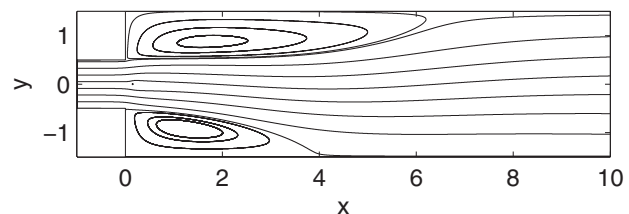

(b)

FIG. 21. Streamlines of the (stable) steady flow in the asymmetric channel $\left(\delta h_{s}=0.05\right)$ (a) without and (b) with control cylinder, placed on the symmetry line of the smaller channel at $x_{0}=0.15$ with $d^{*}=0.02$.

the smaller channel and $d^{*}=0.02$ ). We remind that, without control, Hawa and Rusak ${ }^{9}$ show that the pitchfork bifurcation changes nature as a small asymmetry of the channel is considered, leading to two disconnected branches of equilibrium states, one describing a gradual and stable change with the Reynolds number (branch I), and the other one (branch II) containing a large-amplitude stable state and a small-amplitude unstable state. Branch II appears at a critical Reynolds number $R e_{c a} \simeq 89$.

In the present test, we computed the solution belonging to branch I without control at $R e=100$. As shown in Fig. 21(a), this solution is characterized by a large asymmetry, also due to the asymmetry of the geometry. The control cylinder has been introduced impulsively in this flow; after a transient the flow reaches the steady solution depicted in Fig. 21(b). This controlled state still belongs to branch I, which is now the only possible one since the appearance of branch II is delayed to $R e=115$. Consequently, since the bifurcation is moved to larger Reynolds numbers due to the control, the asymmetry of branch I at $R e=100$ is sensibly reduced, as shown in Fig. 21(b). We remark that the test just described shows that the control behavior remains consistent and effective also when applied in conditions that are far from the design ones.

\section{CONCLUSIONS}

The flow in a 2D symmetric channel with a sudden expansion $(E R=3)$ has been considered here. A linear stability analysis of this problem shows that the symmetric flow configuration becomes unstable for $R e>81.2$. The asymmetric instability has been investigated here with the objective of proposing a passive control strategy to stabilize the symmetric flow.

Two complementary sensitivity analyses have been carried out to characterize the considered instability and to have quantitative indications on how to design the control. The first one gives the sensitivity of the unstable eigenvalue to a forcing term in the linearized equations describing the evolution of small-amplitude perturbations of the base flow. One among the outputs of this analysis is the identification of the core of the instability, carried out by inspecting the spatial structure of the product between the direct and adjoint eigenfunctions associated to the unstable mode. It is shown that the core of the instability is located in two symmetric regions contained inside the two symmetric recirculation zones of the base flow. In the second analysis, we investigated the sensitivity of the eigenvalue to a modification of the base flow. For a generic perturbation of the base flow two symmetric zones of high sensitivity very similar to the ones corresponding to the core of the instability have been observed.

Successively, a realistic control strategy, based on the introduction in the flow of a small circular cylinder, has been investigated first by means of a linear analysis. In this context the cylinder is modeled by the introduction of a localized drag force acting both on the base-flow equations and on the linearized perturbation equations. Consequently, the sensitivity analyses described above have been used to predict the effects of the cylinder on the flow instability. This analysis indicates that the most effective locations for the control cylinder are contained in a region around the centerline near the expansion corners.

The sensitivity analysis used to predict the combination of cylinder position and diameter for the stabilization of the flow is based on the flow equations linearized around the uncontrolled flow, in which the cylinder is mimicked as a localized force. Neglected nonlinear effects due to a finite 
amplitude of the control action have been investigated. In particular, it has been shown that for values of the Reynolds number significantly larger than the critical one $(110 \leq R e \leq 120)$, the flow is not controllable anymore with a single force applied in the position indicated by the linear analysis. This behavior clearly shows the importance of considering the nonlinear effects due to a finite amplitude of the forcing. Moreover, the characterization of the trust region of the linearized analysis, which is partially carried out here, is a necessary preliminary step for the application of iterative strategies to design the passive control, as suggested in Camarri and Iollo. ${ }^{14}$

The effectiveness of the considered control strategy has been verified through DNS in which a real control cylinder is introduced. These simulations validate the strategy followed for the design of the control. Moreover, the capability of the control to recover the target flow starting from far initial conditions and its robustness with respect to a small asymmetry of the diffuser geometry have been assessed. Finally, DNS has been used to provide a physical interpretation for the success of the proposed control. It has been shown that the interaction of vorticity between the wake of the control cylinder and the channel flow is weak, and it is not sufficient to justify the success of the control. Conversely, two possible physical mechanisms have been identified, both consequent to the streamlines modification caused by the drag force exerted by the cylinder. Even if a quantitative analysis of the relative importance of those two factors for the success of the control is difficult, we have proposed a method which allows to verify whether the control remains active or not when the modification in the vorticity production from the walls of the larger channel induced by the control is eliminated. The result is that, without this factor, the flow is not stabilized.

\section{ACKNOWLEDGMENTS}

CASPUR computing center (Roma, Italy) is gratefully acknowledged for allowance of computational resources.

\section{APPENDIX: STABILITY FOR THREE-DIMENSIONAL PERTURBATIONS}

Throughout this paper, we claim that the leading global mode is two-dimensional. To verify this, we reformulate the stability problem (3) for a three-dimensional disturbance flow.

Since the base flow is stationary and homogeneous in the spanwise direction $\mathrm{z}$, the threedimensional perturbations may be looked for as a Fourier mode,

$$
\mathbf{q}^{\prime}(x, y, z, t)=\frac{1}{2}\left\{(\hat{u}, \hat{v}, \hat{w}, \hat{p})(x, y) e^{i k z+\sigma t}+c . c .\right\},
$$

where $k$ is the real spanwise wavenumber, $\sigma$ the complex growth rate and c.c. stands for the complex conjugate of the preceding expression. By substituting this normal mode decomposition into the linearized equations governing the dynamics of the disturbance, we obtained

$$
\left\{\begin{array}{l}
\sigma \hat{\mathbf{u}}+\hat{\mathbf{u}} \cdot \nabla_{k} \mathbf{U}_{b}+\mathbf{U}_{\mathbf{b}} \cdot \nabla_{k} \hat{\mathbf{u}}+\nabla_{k} \hat{p}-\frac{1}{R e} \nabla_{k}^{2} \hat{\mathbf{u}}=\mathbf{0} \\
\nabla_{k} \cdot \hat{\mathbf{u}}=0
\end{array}\right.
$$

where $\nabla_{k}\left(\partial / \partial_{x}, \partial / \partial_{y}, i k\right)$ and $\nabla_{k}^{2}=\nabla_{k} \cdot \nabla_{k}$ are the Fourier-transformed gradient and Laplacian operators, respectively. The above equations are completed by homogeneous boundary conditions on the solid walls and a free-outflow condition at the outlet. Once discretized Eqs. (A2) become a discrete eigenvalue problem. For each value of the transverse wavenumber $k$, the generalized eigenvalue problem is solved. The leading eigenvalue $\sigma_{L}$ is plotted as a function of the transverse wavenumber $k$ on Fig. 22 for the Reynolds number $R e=80$. For each value of the transverse wavenumber, all the eigenmodes are damped and the most unstable mode is the two-dimensional one.

Similar behavior is also found for the flow in the channel with the control cylinder and for the asymmetric base flow. Thus we could conclude that the asymmetric instability is a two-dimensional phenomena. 


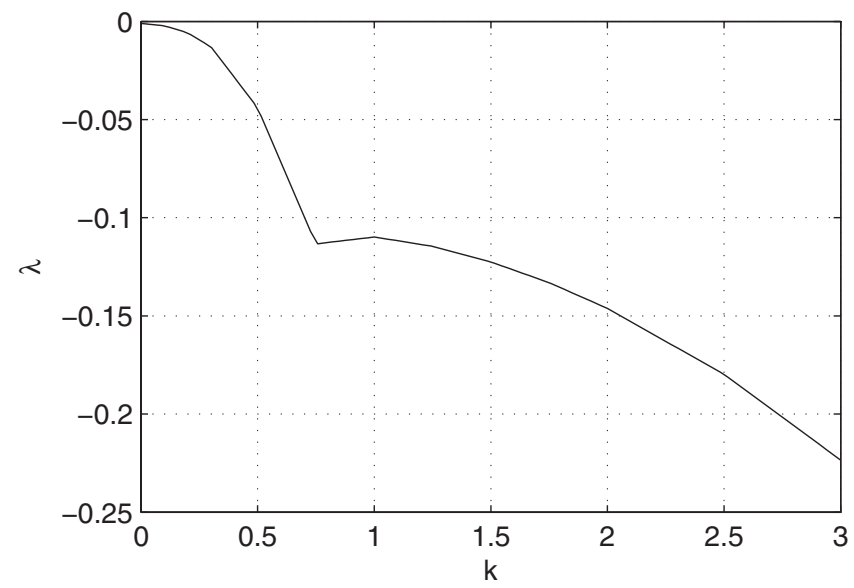

FIG. 22. Temporal growth rate of the leading eigenmode $\sigma_{L}$ (in the present case $\sigma_{L}$ is real) as a function of the transverse wavenumber $k$ at $R e=80$.

${ }^{1}$ F. Durst, A. Melling, and J. Whitelaw, "Low Reynolds number flow over a plane symmetric sudden expansion," J. Fluid Mech. 64, 111-128 (1974).

${ }^{2}$ F. Durst, J. Pereira, and C. Tropea, "The plane symmetric sudden-expansion flow at low Reynolds numbers," J. Fluid Mech. 248, 567-581 (1993).

${ }^{3}$ W. Cherdron, F. Durst, and J. Whitelaw, "Asymmetric flows and instabilities in symmetric ducts with sudden expansions," J. Fluid Mech. 84, 13-31 (1978).

${ }^{4}$ R. Fearn, T. Mullin, and K. Cliffe, "Nonlinear flow phenomena in a symmetric sudden expansion," J. Fluid Mech. 211, 595-608 (1990).

${ }^{5}$ F. Battaglia, S. J. Tavener, A. K. Kulkarni, and C. L. Merkle, "Bifurcation of low Reynolds number flows in symmetric channels," AIAA J. 35, 99-105 (1997).

${ }^{6}$ D. Drikakis, "Bifurcation phenomena in incompressible sudden expansion flows," Phys. Fluids 9, 76 (1997).

${ }^{7}$ T. Hawa and Z. Rusak, "The dynamics of a laminar flow in a symmetric channel with a sudden expansion," J. Fluid Mech. 436, 283-320 (2001).

${ }^{8}$ N. Alleborn, K. Nandakumar, H. Raszillier, and F. Durst, "Further contributions on the two-dimensional flow in a sudden expansion,” J. Fluid Mech. 330, 169-188 (1997).

${ }^{9}$ T. Hawa and Z. Rusak, "Viscous flow in a slightly asymmetric channel with a sudden expansion," Phys. Fluids 12, 2257-2267 (2000).

${ }^{10}$ T. P. Chiang, T. W. H. Sheu, and S. K. Wang, "Side wall effects on the structure of laminar flow over a plane-symmetric sudden expansion," Comput. Fluids 29, 467-492 (2000).

${ }^{11}$ F. Battaglia and G. Papadopoulos, "Bifurcation characteristics of flows in rectangular sudden expansion channels," ASME J. Fluids Eng. 128, 671-679 (2006).

${ }^{12}$ F. Giannetti and P. Luchini, "Structural sensitivity of the first instability of the cylinder wake," J. Fluid Mech. 581, 167-197 (2007).

${ }^{13}$ O. Marquet, D. Sipp, and L. Jacquin, "Sensitivity analysis and passive control of cylinder flow," J. Fluid Mech. 615, 221-252 (2008).

${ }^{14}$ S. Camarri and A. Iollo, "Feedback control of the vortex-shedding instability based on sensitivity analysis," Phys. Fluids 22, 094102 (2010).

${ }^{15}$ ESDU, "Mean forces, pressures and flow field velocities for circular cylindrical structures: Single cylinder with twodimensional flow," ESDU Technical Report 80025, 1986.

${ }^{16}$ C. Taylor and P. Hood, "A numerical solution of the Navier-Stokes equations using the finite element technique," Comput. Fluids 1, 73-100 (1973).

${ }^{17}$ A. Quarteroni, R. Sacco, and F. Saleri, Numerical Mathematics (Springer, Berlin-Heidelberg, 2007).

${ }^{18}$ S. Balay, J. Brown, K. Buschelman, V. Eijkhout, W. D. Gropp, D. Kaushik, M. G. Knepley, L. Curfman McInnes, B. F. Smith, and H. Zhang, "PETSc Users Manual," ANL-95/11 - Revision 3.3, Argonne National Laboratory, 2012.

${ }^{19}$ İ. Dağtekin and M. Ünsal, "Numerical analysis of axisymmetric and planar sudden expansion flows for laminar regime," Int. J. Numer. Methods Fluids 65, 1133-1144 (2011).

${ }^{20}$ A. Revuelta, "On the two-dimensional flow in a sudden expansion with large expansion ratios," Phys. Fluids 17, 028102 (2005). 